\title{
From diffuse damage to strain localization from an Eikonal Non-Local (ENL) Continuum Damage model with evolving internal length
}

\author{
Giuseppe Rastiello $^{\mathrm{a}, *}$, Cédric Giry ${ }^{\mathrm{b}}$, Fabrice Gatuingt ${ }^{\mathrm{b}}$, Rodrigue Desmorat ${ }^{\mathrm{b}}$ \\ ${ }^{a}$ DEN - Service d'études mécaniques et thermiques (SEMT), CEA, Université Paris-Saclay, 91191, Gif-sur-Yvette, France \\ ${ }^{b}$ LMT, ENS Cachan, CNRS, Université Paris-Saclay, 94235, Cachan, France
}

\begin{abstract}
Integral Non-Local (INL) formulations are often used to regularize Continuum Damage computations, in the presence of stress softening for instance. The introduction of a characteristic/internal length allows for avoiding pathological mesh dependency. Some questions concerning the identification of the characteristic length, its possible evolution during damage process and the need for special treatments of non-locality operators near boundaries (e.g. edges, cracks) are however still open. A physical request is that material points separated by a crack (or an highly damaged zone) should not interact. Despite what is done in standard Integral Non-Local theories, this can be obtained by allowing non-local interactions to evolve depending on mechanical fields (e.g. damage, strain, stress). The Eikonal Non-Local (ENL) formulation provides a novel interpretation of damage dependent non-local interactions. Based on the Wentzel-KramersBrillouin (WKB) approximation for high-frequency wave propagation in a damaged medium, this formulation defines the interaction distances as the solution of a stationary damage dependent Eikonal equation. It allows for the modeling of non-local interactions which gradually vanish in damaged zones, thus ensuring a progressive transition from diffuse damage to fracture in a natural way. The numerical implementation and properties of this regularization technique are investigated and discussed. From a numerical viewpoint, a Fast Marching method is used to compute non-local interaction distances between Gauss integration points. Geodesic distances are then used to define the kernel of weighting function to be used in integral non-local averaging. Several numerical results of quasi-statics simulations of quasi-brittle fracture in isotropic media are presented.
\end{abstract}

Keywords: Eikonal Non-Local damage, Regularization, Evolving non-local interactions, Strain Localization, Fast Marching method

DOI: https://doi.org/10.1016/j.cma.2017.12.006

\section{Introduction}

Continuum damage models are widely used to simulate fracture of quasi-brittle materials (e.g. concrete) in reason of their relative simplicity and their ability to handle multi-axial states of stresses. However, from a mathematical viewpoint, it is well known that when the material softens the mechanical problem becomes ill-posed with an infinite number of solutions. From a numerical viewpoint, this gives rise to a pathological sensitivity on the mesh adopted for the spatial discretization of the computed structure.

Different methods exist in the literature in order to regularize the damage evolution. One should cite, among others, integral non-local models (Pijaudier and Bazant, 1987, Pijaudier-Cabot and Benallal, 1993,

\footnotetext{
*e-mail: giuseppe.rastiello@cea.fr
} 
Bažant and Jirásek, 2002, Bažant and Jirásek, 2002), gradient-enhanced models (Frémond and Nedjar, 1996, Peerlings et al., 2001), phase-field models (Miehe et al., 2010) and thick level-set (TLS) methods (Moës et al., 2011, 2014, van der Meer and Sluys, 2015). Integral non-local (INL) formulations (Pijaudier and Bazant, 1987), in particular, are often used due to their strong theoretical background and numerical robustness. Some questions concerning the effect and the formulation of boundary conditions (free edges, notches and preexisting flaws) remain however still open. A main drawback of the standard INL theory consists in nonphysical interactions, through the non-local averaging process, of material points across damaged bands, cracks and holes. Different formulations have been proposed in the literature in order to reduce such kind of secondary/parasite effects. In particular, a local damage evolution along free edges - i.e. with a vanishing characteristic/internal length - was proposed in (Pijaudier-Cabot et al., 2007, Krayani et al., 2009, Pijaudier-Cabot and Dufour, 2010, Bazant et al., 2010). non-local interactions evolving with mechanical fields (e.g., stress, strain, damage) were introduced by different authors (Geers et al., 1998, Pijaudier-Cabot et al., 2004, Simone et al., 2003, Nguyen, 2011, Giry et al., 2011, Saroukhani et al., 2013) in order to model a progressive transition from diffuse damage to strain localization, thus bridging Damage Mechanics and Fracture Mechanics as the non-local interactions vanish.

The recent Eikonal Non-Local (ENL) formulation (Desmorat et al., 2015) provides a novel interpretation of damage dependent evolving non-local interactions, both in isotropic and anisotropic contexts. From a differential geometry viewpoint, this approach leads to consider that damage induces a curvature of the Riemannian space in which interaction distances are computed. From a mathematical point of view, interaction distances between material points are computed as solution of an isotropic time-independent Eikonal equation (a stationary case of the Hamilton-Jacobi equation) with a damage dependent metric function. Geodesic interaction distances computed in the curved space are thus used to computed non-local variables driving the damage evolution, thus preserving the general theoretical framework of INL theories.

Theoretical and uniaxial properties of the ENL framework have been studied in the cited work. Numerical implementation and properties of such a regularization technique are addressed in present work. A numerical formulation for modeling damage dependent non-local interactions within mechanical computations is obtained by coupling a Fast Marching (Sethian, 1996) algorithm - for the computation of the interaction distances - and a standard Finite Element (FE) procedure - for solving the quasi-static equilibrium equations. Attention is focused on isotropic damage models only. The formulation proposed is however general, and can be adapted to more complex constitutive laws such as second order anisotropic damage models (Cordebois and Sidoroff, 1982, Murakami and Ohno, 1978, Fichant et al., 1999, Halm and Dragon, 1998, Lemaitre and Desmorat, 2005, Desmorat et al., 2007, Desmorat, 2016).

The paper is structured in four parts as follows. The theoretical background of Integral and Eikonal NonLocal framework is recalled briefly first. Main aspects of Integral Non-Local regularization techniques are presented. Then, the ENL formulation is introduced and discussed. In the second part of the paper non-local interactions in "artificially" damaged domains are studied (damage fields are fixed a priori, and do not derive from a mechanical computation). For this purpose, the Eikonal equation is solved through a second order accurate Fast Marching algorithm over a regular and orthogonal grid. A Finite Element (FE) implementation of an ENL isotropic damage model is then presented. Real-time coupling of non-linear FE solution procedures and Fast Marching algorithms (for the computation of non-local interaction distances) is described. In the final part of the paper, some numerical examples of quasi-statics simulations of quasi-brittle fracture are given. We explore the capabilities of the proposed framework in simulating a progressive transition from diffuse damage to strain localization and in regularizing damage evolution. Several comparisons with the standard INL formulation are established in terms of global/structural responses and damaging process evolutions in order to illustrate the main features of the proposed model.

\section{Eikonal Non-Local (ENL) damage formulation}

Consider a $n$-dimensional body $\Omega \in \mathbb{R}^{n}$, and suppose that its constituting material behaves according to a scalar isotropic Continuum Damage model with Helmholtz free-energy density:

$$
\psi=\psi(\boldsymbol{\epsilon}, d)=(1-d)\left[\mu \boldsymbol{\epsilon}: \boldsymbol{\epsilon}+\frac{1}{2} \lambda(\operatorname{tr} \boldsymbol{\epsilon})^{2}\right]
$$

Page 2 of 26 
where ":" denotes the double contraction operator between second order tensors, "tr" is the trace operator, $(\lambda, \mu)$ are the homogeneous Lamé parameters, $\boldsymbol{\epsilon}=\boldsymbol{\epsilon}(\boldsymbol{x})$ is the second order strain tensor and $d=d(\boldsymbol{x}) \in[0,1]$ is the scalar damage variable. Under the small strain assumption, $\epsilon$ is the symmetric part of the gradient of the displacement field $\boldsymbol{u}=\boldsymbol{u}(\boldsymbol{x})$. With $\mathbf{I}$ the second order identity tensor, the Cauchy stress tensor $\boldsymbol{\sigma}=\boldsymbol{\sigma}(\boldsymbol{\epsilon}, d)$ and the thermodynamic force $Y=Y(\boldsymbol{\epsilon})$ associated with damage read:

$$
\boldsymbol{\sigma}=\frac{\partial}{\partial \boldsymbol{\epsilon}} \psi=(1-d)[2 \mu \boldsymbol{\epsilon}+\lambda(\operatorname{tr} \boldsymbol{\epsilon}) \mathbf{I}] \quad Y=-\frac{\partial}{\partial d} \psi=\mu \boldsymbol{\epsilon}: \boldsymbol{\epsilon}+\frac{1}{2} \lambda(\operatorname{tr} \boldsymbol{\epsilon})^{2}
$$

The scalar damage variable describes the material degradation process and rises from $d=0$ (sound material) to $d=1$ (completely damaged material). Its evolution is driven by a local variable $w=w(\boldsymbol{x})$, typically assumed for quasi-brittle materials equal to $Y$ (Marigo, 1981) or to an equivalent strain measure $\hat{\epsilon}=\hat{\epsilon}(\boldsymbol{x})$ (e.g. Mazars (1984), de Vree et al. (1995), Ragueneau et al. (2008)):

$$
d=g\left(\max _{t} w\right)
$$

and satisfies the Kuhn-Tucker loading-unloading conditions:

$$
f \leq 0 \quad \dot{\kappa} \geq 0 \quad f \dot{\kappa}=0
$$

where $f=f(w, d, \ldots)$ is the damage criterion function and where the damage multiplier $\kappa$ can be the damage $d$ itself or a - monotonic, positive - function of the damage, $\kappa=g^{-1}(d)$.

\subsection{Integral Non-Local (INL) damage evolution model}

The main idea of INL approaches applied to Continuum Damage Mechanics (Pijaudier and Bazant, 1987) is to make the damage growth of Eqs. (3) and (4) governed by a non-local variable $w^{\mathrm{NL}}=w^{\mathrm{NL}}(\boldsymbol{x})$. The standard INL framework is obtained by weighted averaging of $w(\boldsymbol{x})$ over the domain $\Omega$ :

$$
w^{\mathrm{NL}}\left(\boldsymbol{x}_{x}\right)=\frac{1}{V_{\phi}\left(\boldsymbol{x}_{x}\right)} \int_{\Omega} \phi\left(\xi_{x s}^{\mathrm{INL}}\right) w\left(\boldsymbol{x}_{s}\right) \mathrm{d} V \quad V_{\phi}\left(\boldsymbol{x}_{x}\right)=\int_{\Omega} \phi\left(\xi_{x s}^{\mathrm{INL}}\right) \mathrm{d} V
$$

where $\xi_{x s}^{\mathrm{INL}}=\xi_{x s}^{\mathrm{INL}}\left(\boldsymbol{x}_{x}, \boldsymbol{x}_{s}\right) \in[0,+\infty)$ is the ratio of the Euclidean distance between material points $\boldsymbol{x}_{s}$ and $\boldsymbol{x}_{x}$ to the characteristic/internal length $\ell_{c}$ (considered as a constant material parameter):

$$
\xi_{x s}^{\mathrm{INL}}=\frac{\ell_{x s}}{\ell_{c}}=\frac{\left\|\boldsymbol{x}_{s}-\boldsymbol{x}_{x}\right\|}{\ell_{c}}
$$

and $\phi=\phi(\xi)$ is a positive non-local weighting function. This latter is chosen to be equal to unity when $\xi=0$, monotonically decreasing with respect to $\xi$, and vanishing when $\xi \rightarrow 1$. Typical forms for $\phi(\xi)$ are Gaussian or bell-shaped polynomial functions (Figure 1):

$$
\phi(\xi)=\exp \left(-\xi^{2} / 2\right) \quad \text { or } \quad \phi(\xi)=\left\langle 1-\xi^{2}\right\rangle^{2}
$$

where brackets " $\langle\cdot\rangle$ " denote the Macaulay operator (with $a \in \mathbb{R},\langle a\rangle=\max (0, a)$ ). The bell polynomial function (Bažant and Jirásek, 2002) is often preferred in numerical computations, because it ensures null interactions (i.e., $\phi(\xi)=0$ ) between any pair of material points such that their distance is larger than $\ell_{c}$ (i.e., $\xi \geq 1$ ). This also allows for computing the averaged non-local field (5) by integration over smaller sub-domains:

$$
\mathcal{H}=\mathcal{H}\left(\boldsymbol{x}_{x}\right)=\left\{\boldsymbol{x}_{s} \in \Omega: \ell_{x s} \leq \ell_{c}\right\} \subset \Omega
$$

In the following $\mathcal{H}$ is also called the "non-local interaction domain" or the "non-local horizon" of $\boldsymbol{x}_{x}$.

\subsection{Eikonal Non-Local (ENL) damage evolution model}

According to definition (6), any pairs of material points separated by the same Euclidean distance interact in the same way. A physical request is, however, that material points across cracks, holes and highly damaged zones do not interact (or at least reduce their interactions). 
a)

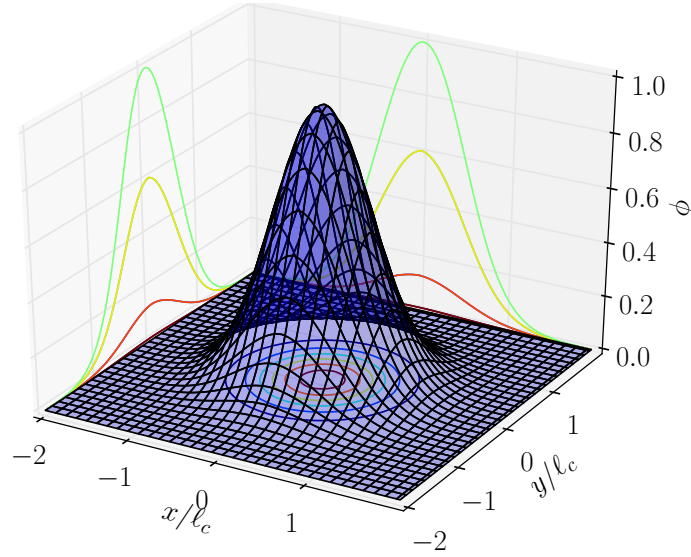

b)

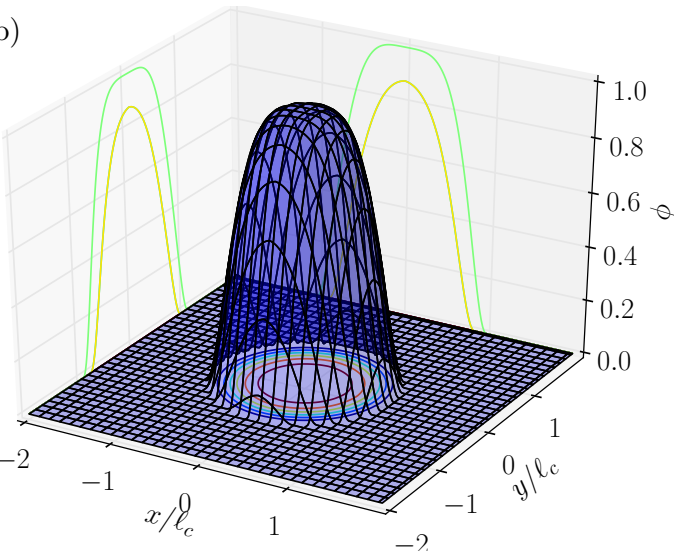

Figure 1: Typical non-local weighting functions: Gaussian (a) and bell-shaped polynomial functions (b)

\subsubsection{From Euclidean distances to propagation time}

Theoretically derived by Desmorat et al. (2015), the ENL formulation models this effect by considering damage dependent non-local interactions. This approach can be seen as a "simplified" version of the Internal Time Non-Local (ITNL) formulation first proposed by Desmorat and Gatuingt (2007) and better studied by Desmorat et al. (2015). Based on the analogy between wave and non-local information propagations within a damaged medium, these authors propose to reformulate the averaging formula (5) in terms of the time ratio:

$$
\xi_{x s}^{\mathrm{ITNL}}=\frac{\tau_{x s}}{\tau_{c}}=\frac{\text { wave propagation time }}{\text { characteristic/internal propagation time }} \geq \xi_{x s}^{\mathrm{INL}}=\frac{\ell_{x s}}{\ell_{c}}
$$

where $\tau_{x s}=\tau_{x s}\left(\boldsymbol{x}_{x}, \boldsymbol{x}_{s}, d(\boldsymbol{x})\right)$ is the wave propagation time from $\boldsymbol{x}_{x}$ to $\boldsymbol{x}_{s}$ on $\Omega$, and $\tau_{c}$ is a characteristic/internal time. This latter can be interpreted as the time needed for an elastic wave to cover a distance $\ell_{c}$ in the sound medium. With $c_{0}$ the information celerity in the virgin material (taken as the wave constant speed), $\tau_{c}$ is proportional to the characteristic length as:

$$
\tau_{c}=\ell_{c} / c_{0}
$$

Using a ITNL damage evolution model in one-dimensional computations is simple, because the wave propagation equation can be solved analitically. Some numerical results were provided in (Desmorat et al., 2015). Implementation in two- and three-dimensional contexts may, however, reveal more complex. In that case, no general analytical solutions for the heterogeneous wave propagation equation are available, but tedious numerical solution procedures are needed for computing propagation times. As an example of this, consider solving the Continuum Damage Mechanics problem through a FE formulation and compute the damage field at the Gauss integration points level. Under these conditions, if the FE mesh comprises $n_{g p}$ integration points, using the ITNL approach leads to solve numerically $n_{g p}$ wave propagation problems at each time step (and/or iteration of the non-linear solution process) to update non-local interaction functions. Due to the bijective nature of the wave propagation problem the number of computations can be reduced. However, the resulting computational cost could rapidly become incompatible with the need of performing large scale computations (e.g. in engineering-oriented applications).

\subsubsection{From propagation time to effective geodesic distances}

The ENL framework provides an easier way to catch wave propagation information. Using a WentzelKramers-Brillouin (WKB) or Liouville-Green (LG) approximation (Orszag and Bender, 1978, Hall, 2013) for high frequency waves propagating within a damaged medium, Desmorat et al. (2015) have shown that determining the propagation time $\tau_{x s}$ from a material point $\boldsymbol{x}_{x}$ to any point $\boldsymbol{x}_{s}$ in $\Omega$ is equivalent to seeking a signed distances field $\tilde{\ell}(\boldsymbol{x}) \in[0,+\infty)$ which approximate the viscosity solution of a time-independent 
Eikonal equation (i.e., a stationary Hamilton-Jacobi equation). Under isotropic damage conditions, this new problem reads:

$$
\left\{\begin{array}{l}
m(\boldsymbol{x})\|\operatorname{grad} \tilde{\ell}(\boldsymbol{x})\|=1 \quad \boldsymbol{x} \in \Omega \\
\tilde{\ell}\left(\boldsymbol{x}_{x}\right)=0
\end{array}\right.
$$

where $m(\boldsymbol{x}) \in(0,1]$ is a positive damage dependent scalar isotropic Riemannian metric function:

$$
m(\boldsymbol{x})=\sqrt{1-d(\boldsymbol{x})}>0 \quad \boldsymbol{x} \in \Omega .
$$

According to (11), the iso-level-set curve $\tilde{\ell}(\boldsymbol{x})=$ cst can be seen as an advancing front with speed $m(\boldsymbol{x})$, and the resulting function $\tilde{\ell}(\boldsymbol{x})$ is a signed distance. Furthermore, when $m(\boldsymbol{x})$ is uniform on $\Omega, \tilde{\ell}(\boldsymbol{x})$ can be interpreted as an Euclidean distances field.

The viscosity solution of (11) can now be obtained numerically using highly efficient algorithms, e.g. Label-correcting methods (Bertsekas, 1993), Sweeping algorithms (Zhao, 2005) and Fast Marching algorithms (Sethian, 1996). The latter family of methods is often used due to its relative theoretical and numerical simplicity.

Once the effective/geodesic lengths field $\tilde{\ell}(\boldsymbol{x})$ is known, the ENL formulation keeps unchanged the averaging process (5) of INL models. The major enhancement with respect this averaging concerns the argument of the weighting function $\phi$, which now is the effective length ratio:

$$
\xi_{x s}^{\mathrm{ENL}}=\frac{\tilde{\ell}_{x s}}{\ell_{c}}=\frac{\text { effective/geodesic distance }}{\text { characteristic/internal length }} \geq \xi_{x s}^{\mathrm{INL}}=\frac{\ell_{x s}}{\ell_{c}}
$$

where $\tilde{\ell}_{x s}=\tilde{\ell}\left(\boldsymbol{x}_{x}, \boldsymbol{x}_{s}, d(\boldsymbol{x})\right) \in[0,+\infty)$ is the effective/geodesic distance between points $\boldsymbol{x}_{s}$ and $\boldsymbol{x}_{x}$. According to (11), effective distances coincide with Euclidean distances (as in the INL approach) in undamaged conditions, and increase progressively when damage occurs. Under some conditions, material points separated by an highly damaged zone (or by a crack) could no longer interact (i.e., $\xi^{\mathrm{ENL}} \rightarrow+\infty$, even through $\left.\xi^{\mathrm{INL}}<1\right)$.

\section{Non-local interactions in two-dimensional damaged domains}

Non-local interactions in two-dimensional (2D) damaged domains are illustrated by some examples. Attention is focused on "artificially" damaged media, in order to illustrate some key features of the ENL formulation. When the damage field is not uniform (i.e., the metric field vary over the domain) the Eikonal equation admits closed form viscosity solutions only under some special conditions. A discussion based on numerical results is thus needed. In the following, effective interaction distances are obtained through a second order accurate Fast Marching algorithm (Sethian, 1996). The role of the Fast Marching grid refinement on properly estimating the geodesic distances field is also put into evidence. As it will be shown later in the paper, this aspect could strongly influence the numerical robustness of the ENL damage formulation.

\subsection{Effective interaction distances computation}

Fast Marching (FM) methods are a family of numerical algorithms that are able to find the viscosity solution of inhomogeneous Eikonal equations. First proposed by Sethian (1996) for following monotonically advancing fronts, they are very similar to the Dijkstra's method (Dijkstra, 1959, Cormen et al., 2001) for finding shortest paths on graphs. Using a gradient descent of the distance function, FM algorithms allow for extracting a good approximation of the shortest geodesic paths in various contexts: Euclidean for $m(\boldsymbol{x})=1$, and a weighted Rienmanian manifold when $m(\boldsymbol{x})$ is not constant over the domain. Many FM formulations were proposed in the literature for dealing with orthogonal and triangular grids, first or higher order approximations, isotropic and anisotropic metric funcions (Kimmel and Sethian, 1998, Sethian, 1999). Highly efficient FM numerical libraries are available in most programming languages and can be used directly in computations. 


\subsubsection{Fast Marching method}

The FM algorithm used in computations is based on a second order upwind approximation of the term $\|\operatorname{grad} \tilde{\ell}(\boldsymbol{x})\|$ over a regular and orthogonal grid of points $\left(G^{\mathrm{FM}}\right)$ :

$$
\begin{aligned}
G^{\mathrm{FM}}=\left\{v=v_{i, j}: \boldsymbol{x}\left(v_{i, j}\right)=\boldsymbol{x}_{i, j}=\left(x_{i}, y_{j}\right), x_{i}=i h, y_{j}=\right. & j h, \\
& i=0,1, \ldots,(n-1), j=0,1, \ldots,(n-1)\}
\end{aligned}
$$

where $v_{i, j}$ denotes grid vertices, $h$ is the constant grid spacing and $n$ is the total number of grid points along $x$ and $y$ axis. The total number of vertices is denoted by $N$. With this spatial discretization, the Eikonal equation is discretized according to Rouy and Tourin (1992):

$$
\max \left\{\mathrm{d}_{i, j}^{-x} \tilde{\ell} ;-\mathrm{d}_{i, j}^{+x} \tilde{\ell} ; 0\right\}^{2}+\max \left\{\mathrm{d}_{i, j}^{-y} \tilde{\ell} ;-\mathrm{d}_{i, j}^{+y} \tilde{\ell} ; 0\right\}^{2}=m_{i, j}^{-2}
$$

where $m_{i, j}=m\left(\boldsymbol{x}_{i, j}\right)=\sqrt{1-d\left(\boldsymbol{x}_{i, j}\right)}$ denotes the discretized metric function at location $(i, j)$ and derivatives approximations read:

$$
\begin{aligned}
\mathrm{d}_{i, j}^{-x} & =\frac{3 \tilde{\ell}_{i, j}-4 \tilde{\ell}_{i-1, j}+\tilde{\ell}_{i-2, j}}{2 h} & \mathrm{~d}_{i, j}^{+x} & =-\frac{3 \tilde{\ell}_{i, j}-4 \tilde{\ell}_{i+1, j}+\tilde{\ell}_{i+2, j}}{2 h} \\
\mathrm{~d}_{i, j}^{-y} & =\frac{3 \tilde{\ell}_{i, j}-4 \tilde{\ell}_{i, j-1}+\tilde{\ell}_{i, j-2}}{2 h} & \mathrm{~d}_{i, j}^{+y} & =-\frac{3 \tilde{\ell}_{i, j}-4 \tilde{\ell}_{i, j+1}+\tilde{\ell}_{i, j+2}}{2 h}
\end{aligned}
$$

For more details about the numerical implementation the interested reader can refer to the cited works.

\subsubsection{Error estimate}

Scheme (15) is stable and consistent. Furthermore it ensures that the solution is found with an order of convergence about unity for any constant metric function $m(\boldsymbol{x})=$ cst. with cst. $\in(0,1]$. To illustrate this aspect, consider a square-shaped domain $\Omega=[-1,1] \times[-1,1]$ and solve the Eikonal problem (11) for finding the Euclidean distances field from the vertex located at $\boldsymbol{x}_{x}=(0,0)$. Figure 2 provides the distances field, the absolute $\left(\ell_{i, j}-\ell_{i, j}^{\text {ref }}\right)$ and relative $\left(\ell_{i, j} / \ell_{i, j}^{\text {ref }}-1\right)$ error maps (with respect to the exact/reference solution) obtained uing first and second order FM algorithms.

Convergence rates can be estimated by representing the global error against the inverse of the grid spacing (Fig. 3a). As it is clear from error maps (Fig. 2), however, FM methods accuracy strongly depends on the orientation of the considered pair of grid points with respect to the main orientation of the grid. Indeed, due to the finite difference approximation of the gradient term, error is very small on vertex belonging to axis $x$ and $y$ and becomes maximum for vertex disposed on the diagonal. Distances computed along this axis could be thus used to define a refinement criterion for using FM algorithms in further numerical computations. Note that absolute errors increase when moving away from the central vertex while the inverse is observed for relative errors. In that case, maximum relative errors are always attained on the nearest grid points to the reference point. For that reason this quantity is used in the following to define such a grid refinement criterion. Figure $3 \mathrm{~b}$ depicts the maximum relative error evolution when moving along the diagonal of the grid $\left(y_{i}=x_{i}\right)$. This representation clearly put in evidence that for a given FM mesh refinement level, the second order algorithm provides lower errors than the linear one. As an example of this, for achieving a relative error of $1 \%$, the linear algorithm requires about 110 grid points along the diagonal, while only 17 grid points can be used with the second order formulation. As the algorithmic complexity of both FM methods is of $O(N \ln N)$ and the resulting CPU times are comparable (Fig. 3c), it is straightforward verifying that the second order algorithm allows reducing computational costs. For that reason the second order scheme is used in the following.

\subsection{Vanishing interactions in damaged zones}

Some examples of geodesic distances computations in damaged media are presented. This allows putting into evidence the role of damage (and/or cracks) on the evolution of non-local interactions within two dimensional domains. In this approach, the crack $\Omega_{c}$ is defined as the set of material points such that 
(a) First order
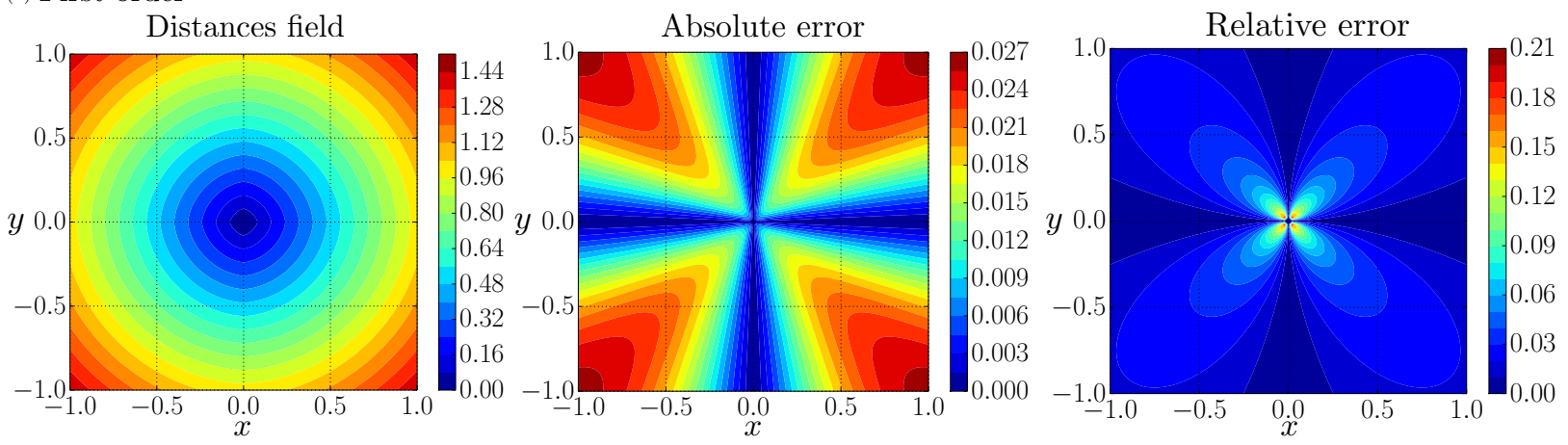

(b) Second order
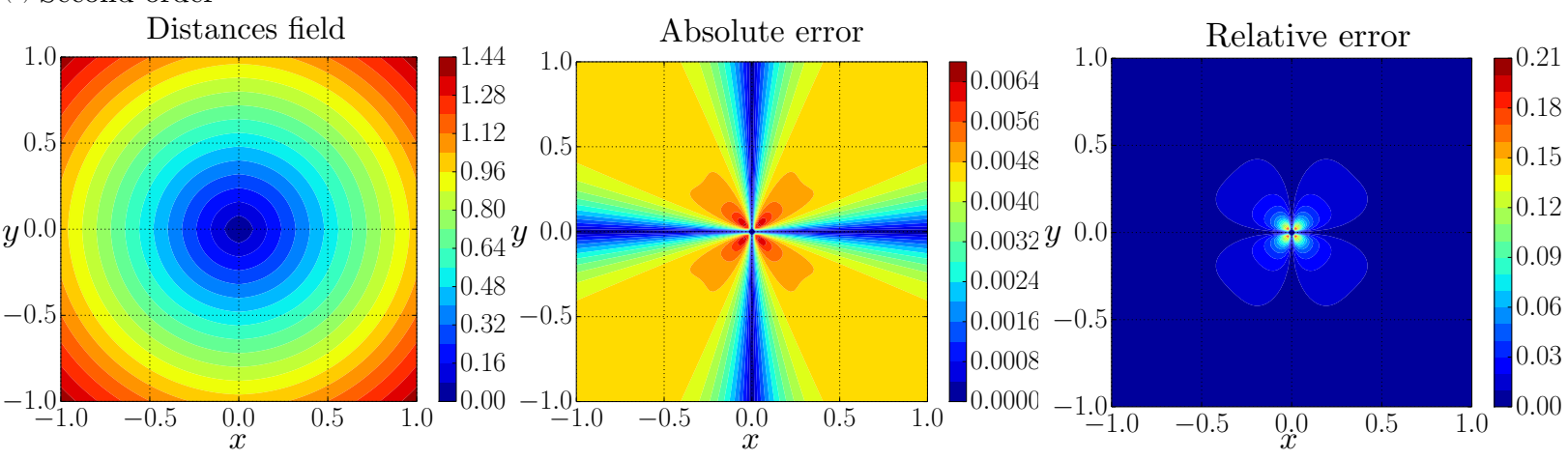

Figure 2: Distances field, absolute and relative error maps computed using first order (a) and second order (b) FM algorithms

damage tends to unity: $\Omega_{c}=\left\{\boldsymbol{x} \in \Omega: d(\boldsymbol{x}) \rightarrow 1^{-}\right\}$. The condition $d=1$ cannot be attained to preserve the positiveness of the front speed $m(\boldsymbol{x})$. However, this does not represent a strong model limitation, as the same constraint derives from the Continuum Damage Mechanics FE formulation. In that case, damage is not allowed to become equal to unity in order to avoid the stiffness matrix to become singular.

\subsubsection{Influence of a sharp crack (localized damage)}

As a first example, the influence of a zero-width crack within a sqaure-shaped domain $\Omega$ is studied. The undamaged state is first considered to define the reference condition: $d(\boldsymbol{x})=0, m(\boldsymbol{x})=1$ on $\Omega$. A cracked domain is then studied. The sharp crack $\Omega_{c}$ is represented by the set of grid points such that $x=0$ and $y \in\left[-\ell_{c}, \ell_{c}\right]$. The corresponding Riemannian metric field takes approximately null values on $\Omega_{c}$ and is Euclidean on $\Omega \backslash \Omega_{c}$.

Figure 4 depicts geodesic distances $\tilde{\ell}(\boldsymbol{x})$ from a reference point $\boldsymbol{x}_{x}=\left(-\ell_{c} / 2,0\right)$ and their influence on the non-local weighting function $\phi(\xi)$ computed for both configurations. A Gaussian distribution was considered for this application, but similar results can be obtained for bell-shaped polynomial functions. Numerical results put in evidence that:

(i) As expected, effective/geodesic and Euclidean distance fields coincides in the undamaged medium: $\tilde{\ell}_{x s}=\ell_{x s}=\left\|\boldsymbol{x}_{s}-\boldsymbol{x}_{x}\right\|$. The non-local weighting function $\phi$ is thus a standard Gaussian bell centered on $\boldsymbol{x}_{x}$, as in the initial INL formulation.

(ii) When the domain is cracked, damage deforms the Riemannian space in which distances are computed. So, provided two material points $\boldsymbol{x}_{x}$ and $\boldsymbol{x}_{s}$ such that the straight line joining them intercepts $\Omega_{c}$, the shortest path between them deviates from the Euclidean setting. Their effective distance thus increases and the resulting non-local interaction reduces. Under some circumstances, this variation is 

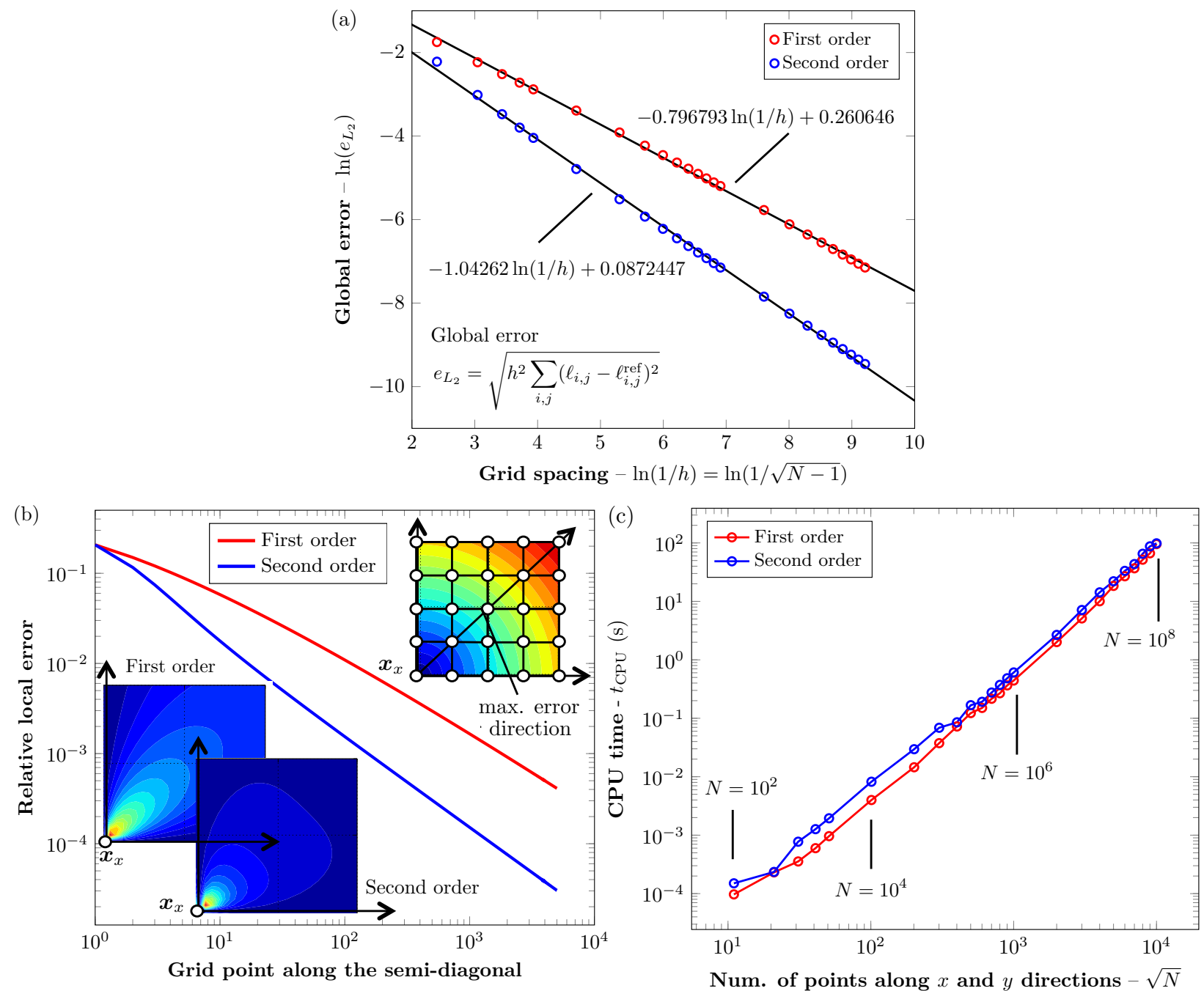

Figure 3: (a) Convergence rate estimation of first and second order FM algorithms, (b) Local relative error evolution along the diagonal of the computational domain, (c) Computational times

such that no interactions occur even though $\left\|\boldsymbol{x}_{s}-\boldsymbol{x}_{x}\right\| \leq \ell_{c}$ (i.e., $\xi_{x s}^{\mathrm{INL}} \leq 1$ ). The non-local weighting function $\phi(\xi)$ thus becomes a truncated Gaussian function.

Similar comments can be made when considering the example (of a notched plate) presented by Desmorat and Gatuingt (2007) for illustrating the ITNL formulation in 2D. The main difference concerns, in that case, the width of the cracked zone $\Omega_{c}$. A comparison between spatial distributions of wave propagation times and geodesic distances (Fig. 5) is however interesting, because it evidences a good qualitative agreement between the spatial distributions of wave propagation times and effective geodesic distances. Note, however, the ENL formulation allows naturally not to consider wave reflections on free edges.

\subsubsection{Influence of a damaged band (diffuse damage)}

The examples considered in previous section illustrated the extreme condition corresponding to a very sharp spatial variation of the damage field. In Continuum Damage Mechanics computations, however, strain localization occurs over a damaged band of finite width. As a consequence, the field $d(\boldsymbol{x})$ will change more smoothly and non-local interactions will also evolve gradually over the domain. To illustrate this situation, a schematic case is considered in Fig. 6 . The body $\Omega$ is crossed vertically by a symmetric damaged band, 

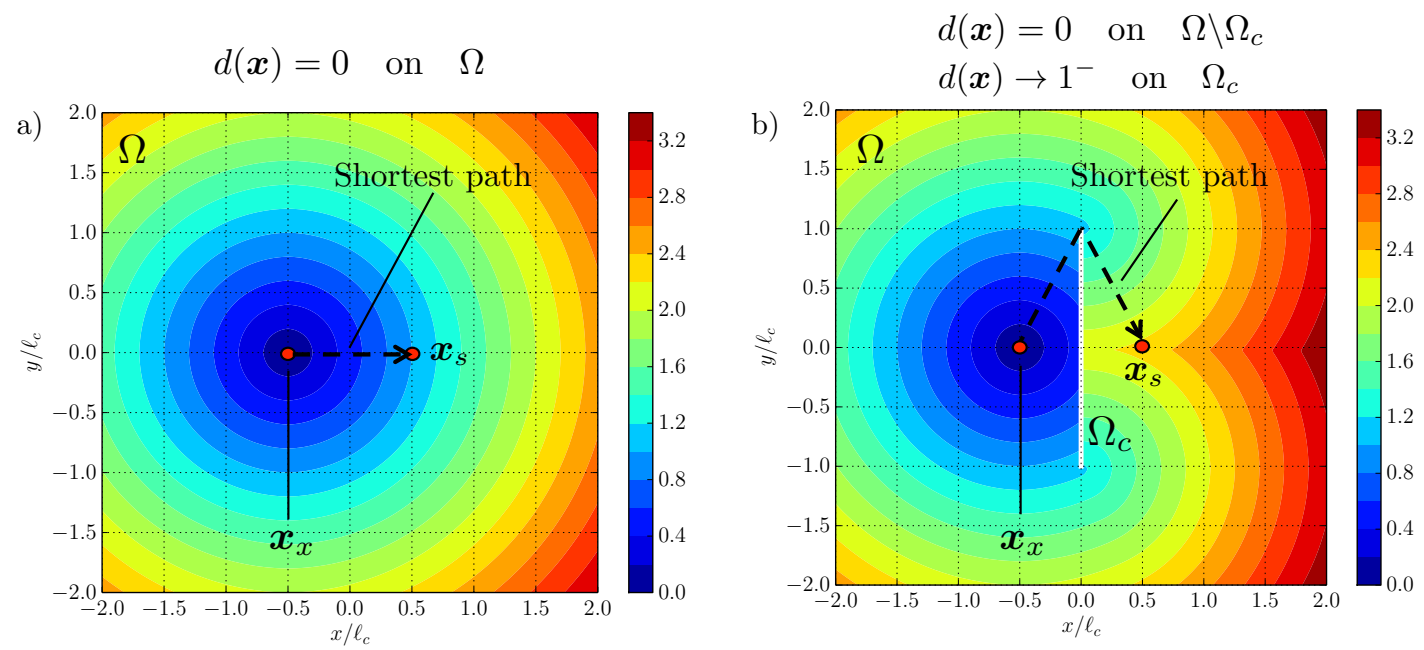

c)

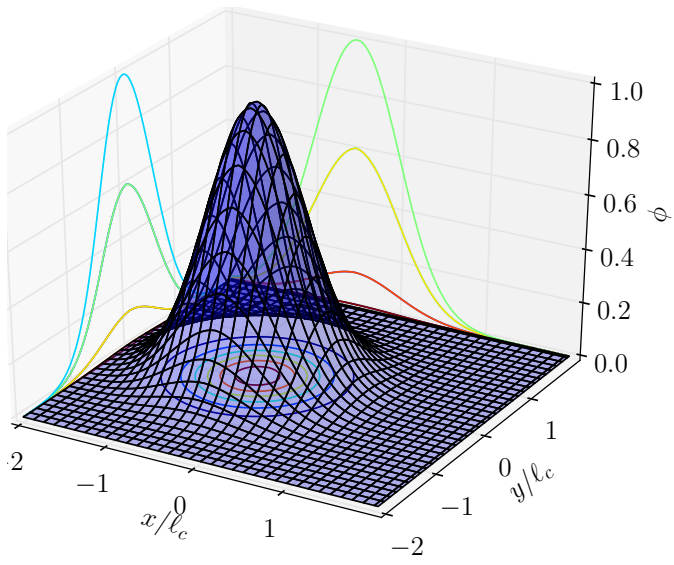

d)

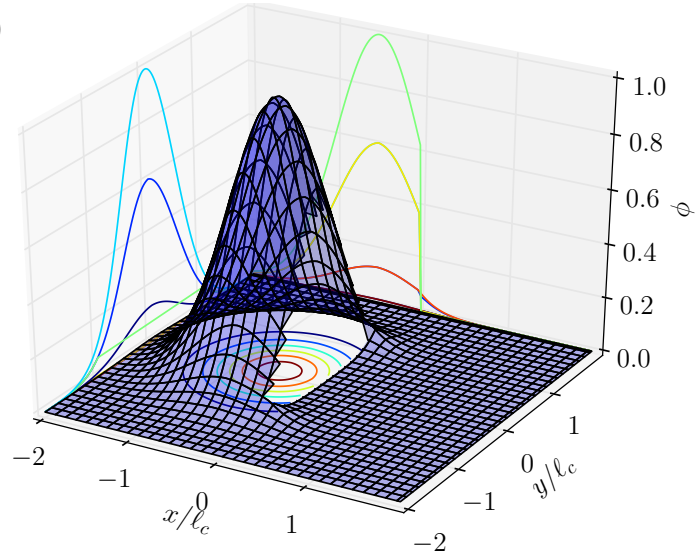

Figure 4: Geodesic distances field $\tilde{\ell}(\boldsymbol{x})$ for a square-shaped domain and its influence on the Gaussian weighting function $\phi(\xi)$ centered on the point $\boldsymbol{x}_{x}$ : (a-c) undamaged medium $\left(m(\boldsymbol{x})=1\right.$ everywhere); (b-d) cracked medium $\left(m(\boldsymbol{x})=1\right.$ on $\Omega \backslash \Omega_{c}$ and $m(\boldsymbol{x})=10^{-5}$ on $\left.\Omega_{c}\right)$. The computational domain is discretized through a regular orthogonal grid comprising $10^{\prime} 201=101 \times 101$ vertex (grid spacing $h=4 \ell_{c} / 100$ )

such that $d(\boldsymbol{x}) \rightarrow 1^{-}$on its symmetry axis and vanishes at a distance $L_{b}=\ell_{c}$ from it (i.e., the width of the damaged band is $\left.2 L_{b}=2 \ell_{c}\right)$. Effective non-local interaction distance fields $\tilde{\ell}(\boldsymbol{x})$ and the co corresponding weighting functions $\phi(\xi)$ are computed for three material points $\boldsymbol{x}_{x}$ placed at different distances from the symmetry axis of the damaged band. As shown in Fig. 6, damage strongly influences non-local interactions within the medium. In particular:

(i) For null or small damage levels, non-local interactions are mainly controlled by Euclidean distances.

(ii) When the damage level increases, non-local interactions reduce and the non-local weighting function progressively loss its symmetry. When a point belonging to the damaged band is considered, this effect is coupled with a progressive increase of non-local interaction distances in all directions. In terms of the weighting function, this corresponds to a progressive reduction of the bell width too.

(iii) If the damage level at the point considered is large enough, no non-local interactions occur. As a consequence the bell width could tend to zero.

This illustrates an expected key feature of the ENL formulation: when it is applied to the regularization of damage evolution models, a progressive transition from a INL damage evolution (in weakly damaged zones) to a completely local damage evolution (in highly damaged zones) can be represented naturally. 


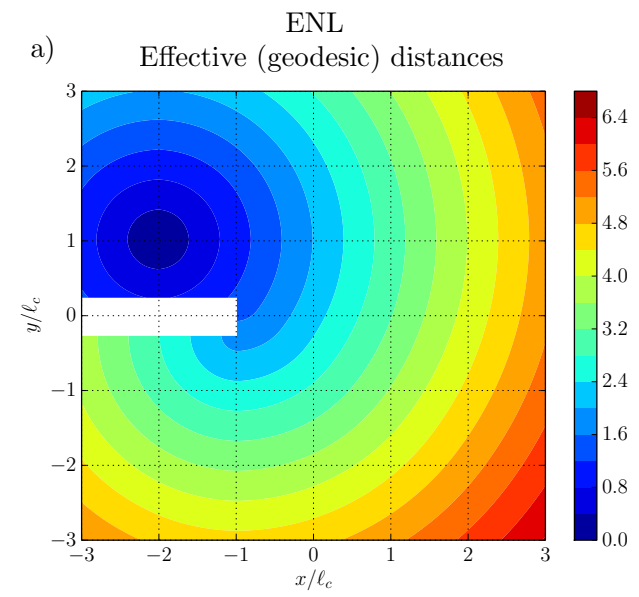

b)

ITNL

Wave propagation time

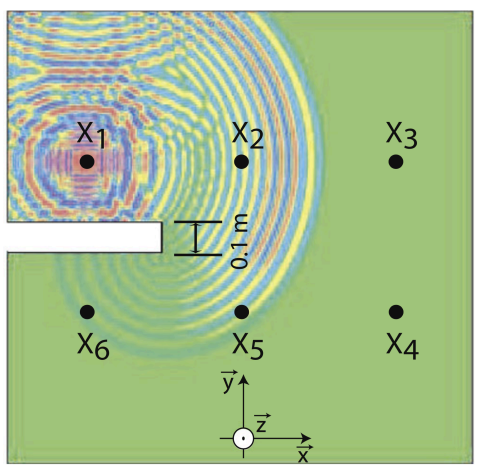

Figure 5: Comparison among effective geodesic distance (a) and wave propagation time (b) fields in a notched square plate

\section{Eikonal Non-Local numerical formulation in the FEM context}

A non-intrusive (or less intrusive as possible) numerical scheme for using ENL isotropic damage models in non-linear Finite Element (FE) computations is presented. Quasi-static equilibrium equations are solved according to a standard FE formulation and an explicit algorithm is used to compute the damage field at the Gauss integration points level. Damage dependent interaction distances between integration points are evaluated using a FM algorithm. For this purpose independent FM grids are defined Gauss point by Gauss point and the metric function is obtained after projection of the damage field from the FE mesh to FM grids. Evolving geodesic distances are finally used to update the non-local field driving damage evolution.

\subsection{Boundary value problem}

Consider a solid body occupying all points of a continuous domain $\Omega$. Its boundary $\partial \Omega$ comprises two non overlapping sub-domains $\partial_{u} \Omega$ and $\partial_{t} \Omega$, such that displacements (Dirichlet boundary conditions) are imposed on $\partial_{u} \Omega$ and loadings (Neumann boundary conditions) on $\partial_{t} \Omega$. The material which constitutes $\Omega$ is supposed to behave according to an isotropic damage model with a single (scalar) damage variable $d$ (see Sec. 1). Quasi-static conditions, in the absence of body forces, are considered and strains are assumed small. Under these conditions, one looks (at any time $t$ ) for an admissible displacement field $\boldsymbol{u}=\boldsymbol{u}(\boldsymbol{x}) \in \mathcal{U}=$ $\left\{\boldsymbol{u}^{*}\right.$ regular enough : $\boldsymbol{u}^{*}=\boldsymbol{u}_{\mathrm{imp}}$ on $\left.\partial_{u} \Omega\right\}$ which solve the differential problem:

$$
\begin{cases}\boldsymbol{u} \in \mathcal{U} & \text { on } \Omega \\ \operatorname{div} \boldsymbol{\sigma}=0 & \text { on } \Omega \\ \boldsymbol{\sigma}=(1-d)(2 \mu \boldsymbol{\epsilon}+\lambda \operatorname{tr} \boldsymbol{1} \mathbf{1}) & \text { on } \Omega \\ d=g\left(\max _{t} w^{\mathrm{NL}}\right) \in[0,1] & \text { on } \Omega \\ \boldsymbol{\epsilon}=\operatorname{Sym}(\operatorname{grad} \boldsymbol{u}) & \text { on } \partial_{u} \Omega \\ \boldsymbol{u}=\boldsymbol{u}_{\mathrm{imp}} & \text { on } \partial_{t} \Omega \\ \boldsymbol{\sigma} \boldsymbol{n}=\boldsymbol{t}_{\mathrm{imp}} & \end{cases}
$$

where "div" denotes the divergence operator, $\boldsymbol{n}$ is the outward normal vector to the boundary $\partial_{t} \Omega, \boldsymbol{t}_{\text {imp }}$ denotes imposed tractions and $\boldsymbol{u}_{\mathrm{imp}}$ stands for imposed displacements.

\subsection{FEM formulation}

Problem (17) is solved numerically using a standard non-linear FE formulation (Zienkiewicz and Taylor, 2000 , Hughes, 2000). For this purpose, the domain $\Omega$ is discretized through a FE mesh $\left(M^{\mathrm{FE}}\right) \operatorname{comprising} n_{\mathrm{el}}$ 
a)
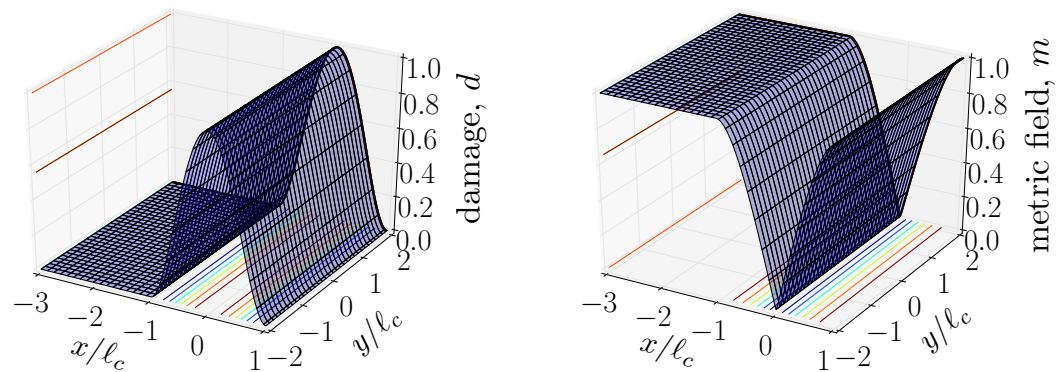

b) Geodesic interaction distances
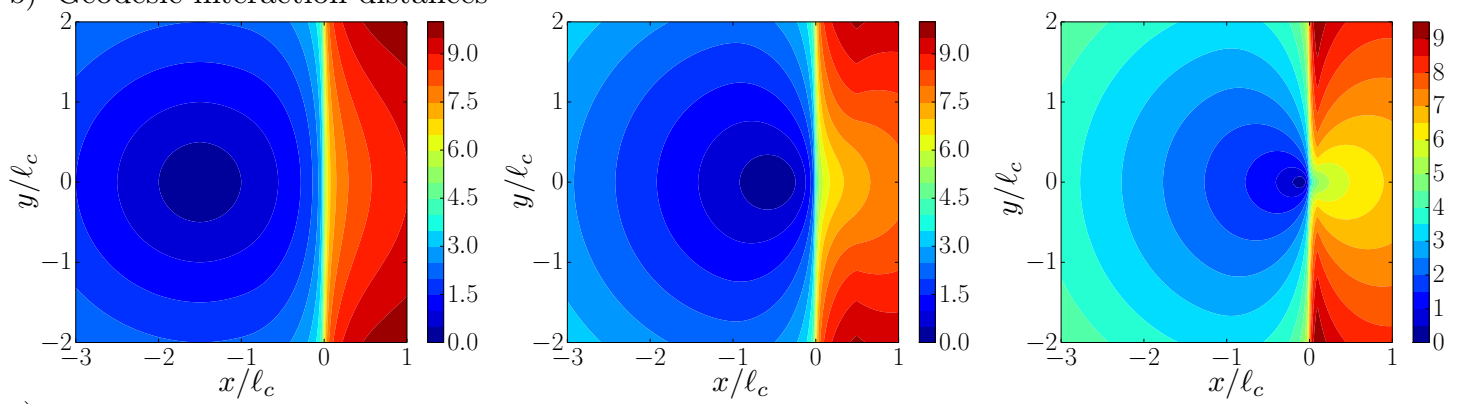

c) Gaussian nonlocal interaction functions
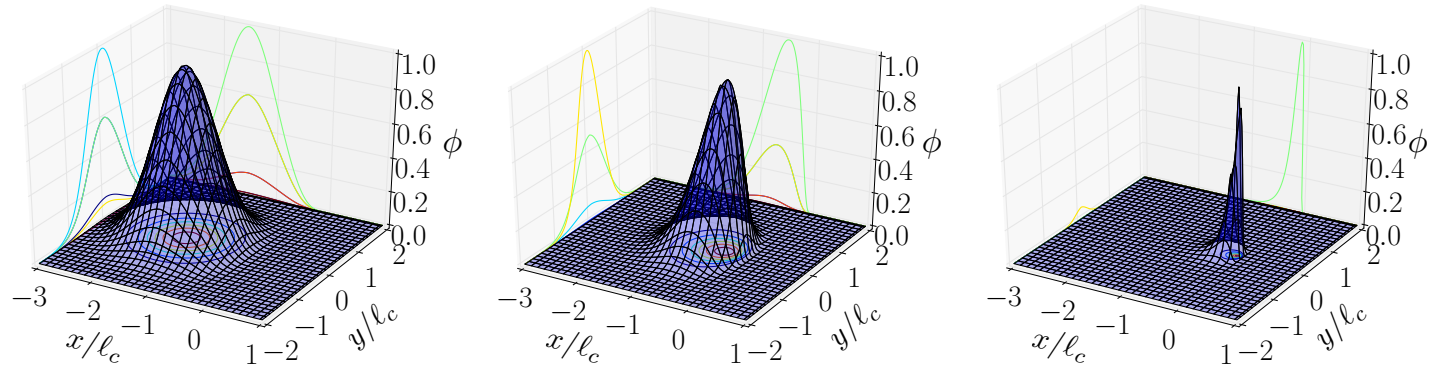

Figure 6: (a) Schematic representation of the damaged band and corresponding metric function $m(\boldsymbol{x})(d(\boldsymbol{x})$ follows a second order polynomial function in the $x$ axis direction and is uniform along $y$ ); (b) geodesic distances fields for three material points $\boldsymbol{x}_{x}$ placed at different distances $\left(1.75 \ell_{c}, 0.75 \ell_{c}\right.$ and $\left.0.075 \ell_{c}\right)$ from the damaged band; and (c) influence on the Gaussian weighting function

iso-parametric finite elements (denoted by $\Omega_{e}$ ). The external load is discretized in pseudo-time according to an ordered sequence of instants $\left\{t_{0}, t_{1}, \ldots, T\right\}$ such that $t_{n+1}>t_{n} \in \mathbb{R}^{+} \cup\{0\}$ for any $n \in \mathbb{N}=\{0,1,2, \ldots\}$. So, provided the nodal displacement and damage fields at loading step $t_{n}$, the solution at step $t_{n+1}$ is searched iteratively through a secant algorithm. In the following, down-script $n+1$ is omitted for sake of compactness. As a consequence, every quantity without down-script should be intended as referred to instant $t_{n+1}$.

\subsubsection{Eikonal Non-Local damage integration}

Elementary damaged stiffness matrices are evaluated at the Gauss points level using an explicit procedure. Given the Gauss integration point occupying the position $\boldsymbol{x}_{x}$, the damage value $d^{k+1}\left(\boldsymbol{x}_{x}\right)$ at the global iteration $k+1$ is computed as:

$$
d^{k+1}\left(\boldsymbol{x}_{x}\right)=\max \left(g\left(w^{\mathrm{NL}, k}\left(\boldsymbol{x}_{x}\right)\right), d_{n}\left(\boldsymbol{x}_{x}\right)\right)
$$

where $w^{\mathrm{NL}, k}\left(\boldsymbol{x}_{x}\right)$ is computed from the solution at iteration $k$.

In INL formulations, $w^{\mathrm{NL}, k}\left(\boldsymbol{x}_{x}\right)$ is computed by directly applying the averaging formula (5) with $\xi_{x s}=$ $\xi_{x s}^{\mathrm{INL}}$. In that case, non-local interactions occurring between Gauss points are controlled by Euclidean distances between them. So, once these information are properly computed (from nodal coordinates) and 
stored, they can be used throughout the solution process without requiring further computations. Conversely, in ENL damage evolution models, non-local interactions change following damage evolution. The resulting numerical implementation is therefore slightly more complex. A possible choice is to recompute interaction distances between $\boldsymbol{x}_{x}$ and its neighbors by solving the Eikonal problem:

$$
\begin{cases}m_{n}(\boldsymbol{x})\left\|\operatorname{grad} \tilde{\ell}_{n}(\boldsymbol{x})\right\|=1 & \boldsymbol{x} \in \mathcal{H} \\ m_{n}(\boldsymbol{x})=\sqrt{1-d_{n}(\boldsymbol{x})} & \boldsymbol{x} \in \mathcal{H} \\ \tilde{\ell}_{n}\left(\boldsymbol{x}_{x}\right)=0 & \end{cases}
$$

at the end of each loading step $t_{n}$, and assume them constant throughout iterations until convergence at step $t_{n+1}$. This allows for avoiding spurious solution oscillations associated with damage distributions which do not correspond to any converged solution (i.e., do not respect the global equilibrium).

It should be noted that, due to the positiveness of the damage rate, the rate of variation of the metric function is always null or negative, i.e., $\Delta m(\boldsymbol{x})=m(\boldsymbol{x})-m_{n}(\boldsymbol{x}) \leq 0$. As a consequence of this, geodesic distances can only increase or remain constant:

$$
\Delta \tilde{\ell}_{x s}=\tilde{\ell}_{x s}-\tilde{\ell}_{x s, n} \geq 0 \quad \forall \boldsymbol{x}_{s} \in \mathcal{H}
$$

and non-local interactions can only reduce or remain constant:

$$
\Delta \phi\left(\xi_{x s}^{\mathrm{ENL}}\right)=\phi\left(\xi_{x s}^{\mathrm{ENL}}\right)-\phi\left(\xi_{x s, n}^{\mathrm{ENL}}\right) \leq 0 \quad \forall \boldsymbol{x}_{s} \in \mathcal{H}
$$

\subsubsection{Geodesic distances computation}

The second order FM algorithm illustrated in Sec. 3.1.1 is applied Gauss integration point by Gauss integration point. For this purpose, regular orthogonal grids $G^{\mathrm{FM}}=G^{\mathrm{FM}}\left(\boldsymbol{x}_{x}\right)$ are defined separately for each Gauss point $\boldsymbol{x}_{x}$. They are $2 \ell_{c} \times 2 \ell_{c}$ in size, and such that central vertex have the same global coordinates $\left(x_{x}, y_{x}\right)$ of $\boldsymbol{x}_{x}:$

$$
\begin{aligned}
G^{\mathrm{FM}}=\left\{v: \boldsymbol{x}(v)=\left(x_{i}, y_{j}\right), x_{i}=x_{x}-\ell_{c}+i h^{\mathrm{FM}}, y_{j}\right. & =y_{x}-\ell_{c}+j h^{\mathrm{FM}}, \\
i & \left.=0,1, \ldots,\left(n^{\mathrm{FM}}-1\right), j=0,1, \ldots,\left(n^{\mathrm{FM}}-1\right)\right\}
\end{aligned}
$$

where $h^{\mathrm{FM}}=h^{\mathrm{FM}}\left(\boldsymbol{x}_{x}\right)$ and $n^{\mathrm{FM}}=n^{\mathrm{FM}}\left(\boldsymbol{x}_{x}\right)$. This ensures reducing useless geodesic distance computations between $\boldsymbol{x}_{x}$ and any integration point $\boldsymbol{x}_{s} \notin \mathcal{H}$. Furthermore, separately applying the FM algorithm for each integration point has the following advantages:

(i) As the metric function depends on the damage field at time step $t_{n}$, Eikonal problems (19) can be solved for each integration point in a parallel fashion;

(ii) FM grids with different refinement levels can be used depending on their position on the FE mesh. Indeed, grid spacing $h^{\mathrm{FM}}$ should be chosen to ensure an accurate estimation of geodesic distances between $\boldsymbol{x}_{x}$ and the nearest Gauss point to it (at least in Euclidean context). Since distances between Gauss points depend on FEs size, one can therefore use denser FM grids in zones where the FE mesh is denser, and to reduce FM grid spacing otherwise.

Based on considerations provided in previous sections concerning orientation dependent errors with standard FM methods, the maximum grid spacing to be used in computations is estimated as:

$$
h^{\mathrm{FM}, \max }=h^{\mathrm{FM}, \max }\left(\boldsymbol{x}_{x}\right)=\frac{1}{\rho} \frac{\sqrt{2}}{2} \ell_{x s}^{\min } \quad \ell_{x s}^{\min }=\ell_{x s}^{\min }\left(\boldsymbol{x}_{x}\right)=\min _{\boldsymbol{x}_{s} \in \mathcal{H} \backslash \boldsymbol{x}_{x}}\left\{\left\|\boldsymbol{x}_{s}-\boldsymbol{x}_{x}\right\|\right\}
$$

where coefficient $\sqrt{2} / 2$ accounts for the observation that the most critical condition is attained when the distance vector $\boldsymbol{x}_{s}-\boldsymbol{x}_{x}$ is parallel to the diagonals of the FM grid, and $\rho \in \mathbb{N}$ is a user defined "grid refinement factor". This latter can be seen as the minimum number of grid points needed for numerically computing the Euclidean distance $\ell_{x s, \min }$ with a fixed error. 
For the second order FM method presented above, choosing $\rho \geq 17$ ensures that the relative numerical error in Euclidean distances computation remains lower than 1\%. This criterion also ensures that INL and ENL formulations lead to the same non-local fields when undamaged conditions are considered. Other criteria for defining this parameter can be of course adopted. However, as such a choice depends on the chosen FM algorithm, this aspect is not further detailed in this paper.

\subsection{Implementation aspects}

The ENL formulation was implemented in a Object Oriented fashion. The "PyFEM" code by de Borst et al. (2012) was used as a starting point for developments. Without going into details of the programming aspects of the numerical implementation, its main ingredients are summarized in the following. Data structures needed for using general INL regularization techniques are defined first. Some special structures for ENL formulations are introduced then.

Initialization phase. For each Gauss integration point $\boldsymbol{x}_{x}$ of each finite element $\Omega_{e}$ :

(i) The discretized non-local interaction domain $\mathcal{H} \in M^{\mathrm{FE}}$ is defined first. For each Gauss point $\boldsymbol{x}_{s} \in \mathcal{H}$, the following information are stored: its identifier and the corresponding element, its global coordinates $\left(x_{s}, y_{s}\right)$, and the Euclidean distance $\ell_{x s}$. These are the only information needed when using INL regularization techniques (in that case, the subsequent steps are not necessary);

(ii) A structured and orthogonal grid $G^{\mathrm{FM}}$ is then defined. According to (23), the total number $\left(n^{\mathrm{FM}}\right)$ of vertex along $x$ and $y$ axis is computed as the smallest odd natural number such that:

$$
n^{\mathrm{FM}}=n^{\mathrm{FM}}\left(\boldsymbol{x}_{x}\right) \geq \min \left\{p \in \mathbb{N}, p \text { odd }: p \geq \frac{2 \ell_{c}}{h^{\mathrm{FM}, \max }}\right\}
$$

This ensures that the central vertex of $G^{\mathrm{FM}}$ has exactly the same global coordinates as the reference Gauss point, $\boldsymbol{x}\left(v_{i, i}\right)=\boldsymbol{x}_{x}$ with $i=\left(n^{\mathrm{FM}}-1\right) / 2$, and that grid spacing is lower than the maximum admissible value, $h^{\mathrm{FM}} \leq h^{\mathrm{FM}, \max }$.

(iii) The metric function is initialized as: $m(v)=m_{0}(v) \rightarrow 0^{+} \forall v \in G^{\mathrm{FM}}$.

(iv) For each grid point $v \in G^{\mathrm{FM}}$, the finite element $\Omega_{e} \in \mathcal{H}$ such that $\boldsymbol{x}(v) \in \Omega_{e}$ is found. Then, the nearest Gauss point $\boldsymbol{x}_{s} \in \Omega_{e}$ to $v$ is computed. It is denoted by $\boldsymbol{x}_{s \rightarrow v}$. This information is used during computations to build Riemannian metric fields from damage values computed at the Gauss points level. No information are stored for grid vertices outside the computational domain (i.e., grid points which do not belong to any finite element).

(v) Finally, for each destination Gauss point $\boldsymbol{x}_{s} \in \mathcal{H}$, the nearest vertex $v \in G^{\mathrm{FM}}$ is found. Its position is denoted by $\boldsymbol{x}_{v \rightarrow s}$. This information is used during computations for transferring effective non-local distances information from the FM grid to the FE mesh.

Once these information are properly stored, the non-linear FE solution process starts.

Current time station $\left(t_{n} \rightarrow t_{n+1}\right)$. At the beginning of the time increment $\left[t_{n}, t_{n+1}\right]$, the solution $\left(\boldsymbol{u}_{n}, d_{n}\right)$ is known. So, for each Gauss point $\boldsymbol{x}_{x}$ of each finite element $\Omega_{e}$ :

(i) The damage value associated to each vertex $v \in G^{\mathrm{FM}}$ is obtained as $d_{n}(v)=d_{n}\left(\boldsymbol{x}_{s \rightarrow v}\right)$. This allows for defining the discretized metric function as $m_{n}(v)=\sqrt{1-d_{n}(v)}>0$, while for vertices outside the computational domain (i.e., not belonging to any $\Omega_{e}$ ) the metric function is maintained at the initialization value, $m_{n}(v)=m_{0}(v)=0$. This condition ensures that for these grid points, computed geodesic distances always tend to infinity. As a consequence, no shortest geodesic paths passing outside the computational domain can exist.

(ii) The geodesic distances field from the source vertex $v_{x, x}$ to any other destination vertex $v \in G^{\mathrm{FM}}\left(\boldsymbol{x}_{x}\right)$ is then computed. 

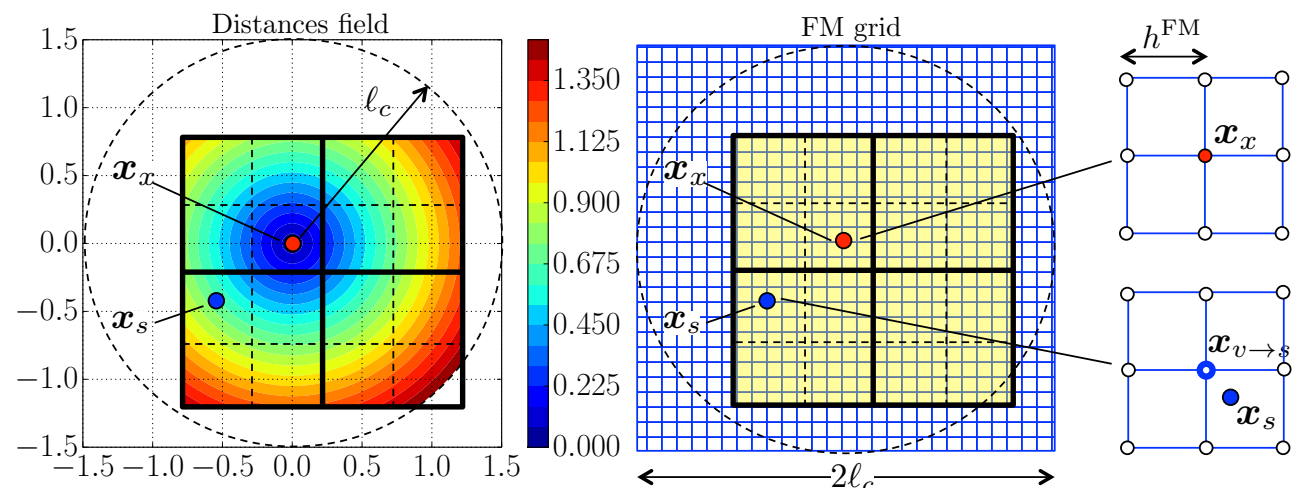

Figure 7: Schematic illustration of the considered quantities for introducing damage dependent non-local interactions within a finite element computation. The FE mesh comprises four square finite elements $L=1$ in size while the internal length is $\ell_{c}=1.5$

(iii) Non-local interaction distances between $\boldsymbol{x}_{x}$ and Gauss points $\boldsymbol{x}_{s} \in \mathcal{H}$ are computed finally. For this purpose we assume that $\tilde{\ell}_{x s, n}=\tilde{\ell}\left(\boldsymbol{x}_{v \rightarrow s}\right)$, even through $\boldsymbol{x}\left(v_{s}\right)$ does not exactly coincides with $\boldsymbol{x}_{s}$. As, in general, highly refined FM grids are used in computations, such a simplified approach provides very good estimates of geodesic distances. A slightly more complex formulation could be obtained, for instance, by finding the three/four nearest FM vertex to $\boldsymbol{x}_{s}$ and computing $\tilde{\ell}_{x s}$ as the linear combination of their geodesic distances with respect to $\boldsymbol{x}_{x}$. This can be achieved easily by considering a sort of "virtual" finite element having these vertices as nodal support, and using shape functions for approximating the effective distances field on $\boldsymbol{x}_{s}$.

(iv) Non-local weighting functions $\phi(\xi)$ are finally updated through the effective length ratio:

$$
\xi_{x s, n}^{\mathrm{ENL}}=\tilde{\ell}_{x s, n} / \ell_{c} \quad \forall \boldsymbol{x}_{s} \in \mathcal{H}
$$

These information are stored at the level of the Gauss point $\boldsymbol{x}_{x}$. So, each Gauss point "knows" its neighbors and the corresponding interaction weights.

\section{Strain localization examples}

Results of quasi-static strain localization in 2D quasi-brittle continua are presented. Attention is focused on simple cases showing the main features of the ENL formulation for modeling damage dependent interactions. For this purpose, strain localization into a tie-specimen submitted to a tensile loading is considered first. This allows validating the numerical formulation and studying its properties. A wedge splitting test is then simulated in order to show some further features of the ENL method.

\subsection{Damage model adopted in computations}

In computations, the non-local damage criterion function is defined in the non-local equivalent strains $\left(\hat{\epsilon}^{\mathrm{NL}}\right)$ space as:

$$
f^{\mathrm{NL}}=f\left(\hat{\epsilon}^{\mathrm{NL}}, \kappa\right)=\hat{\epsilon}^{\mathrm{NL}}-\kappa, \quad \kappa=\max _{t} \hat{\epsilon}^{\mathrm{NL}}
$$

where $\hat{\epsilon}$ is computed according to the definition proposed by Mazars (1984), $\hat{\epsilon}=\sqrt{\left\langle\epsilon_{1}\right\rangle^{2}+\left\langle\epsilon_{2}\right\rangle^{2}}$. In previous equation, $\epsilon_{i}(i=1,2)$ denotes the $i$ th principal strain component and positive parts $\left\langle\epsilon_{i}\right\rangle$ are the extensions. Accordingly, the internal variable $\kappa$ can be interpreted as an equivalent non-local deformation level. It starts at a (damage) threshold level $\kappa_{0}=\epsilon_{0}^{\mathrm{NL}}$ and is updated by requiring that $f^{\mathrm{NL}}=0$ during damage growth, while $\dot{\kappa}=0$ at unloading and when $f^{\mathrm{NL}}<0$. Damage growth is finally supposed to follow the exponential evolution law:

$$
d=g(\kappa)=1-\frac{\kappa_{0}}{\kappa} \exp \left[-\frac{\left\langle\kappa-\kappa_{0}\right\rangle}{\kappa_{c}-\kappa_{0}}\right]
$$

Page 14 of 26 
where $\kappa_{c}=\epsilon_{c}^{\mathrm{NL}}>\epsilon_{0}^{\mathrm{NL}}$ is a material parameter controlling the shape of the damage evolution function. It should be noted that (26) is a simple modeling assumption and that the proposed ENL formulation can be applied without modifications to different non-local damage criteria.

\subsection{Tie-specimen under tensile loading}

Consider a $2 \mathrm{D}$ domain $\Omega$ representing a tie-specimen $L=100 \mathrm{~mm}$ in length (oriented along the $x$-axis) and $W=L / 20=5 \mathrm{~mm}$ in width (along the $y$-axis) submitted to a tensile loading. Boundary and loading conditions are provided in Fig. 8. The domain is discretized through a FE mesh comprising $n_{e l}$ linear quadrangular FEs. Different FE mesh sizes are considered in order to study the numerical regularization properties of the ENL approach. Constitutive model parameters are assigned as follows: $E=100 \mathrm{MPa}$ (Young's modulus), $\nu=0, \kappa_{0}=0.0001, \kappa_{c}=10 \times \kappa_{0}=0.001$ and $\ell_{c}=20 \mathrm{~mm}$ (Lamé constants can be easily computed from $E$ and $\nu$ ).

Calculations are performed assuming plane strains conditions and considering the local, INL and ENL damage evolution models. Strain localization is forced on the center of the specimen by introducing a defect, a weak finite element. This is obtained by slightly reducing its Young modulus with respect to other elements $\left(E_{\text {weak }}=0.9 \times E=90 \mathrm{MPa}\right)$. In order to follow mechanical instabilities (i.e., snap-backs) occurring in the post-peak phase of load, the external load is controlled indirectly through an arc-length type algorithm. Imposed force increments are adapted over loading steps in order to ensure a constant rate of variation of the mean relative horizontal displacement of two pairs of nodes aligned vertically and symmetrically placed $( \pm L / 20)$ with respect of the vertical symmetry axis of the specimen.

\subsubsection{Eikonal Non-Local, Integral Non-Local and local damage models}

The structural response of the sample is represented through the imposed external force and total sample elongation. Figure 8 compares representative responses computed considering the local, ENL and INL damage models for a FE mesh comprising $51=51 \times 1$ elements. In order to follow the evolution of nonlocal interactions inside the damaged band, geodesic distances computed from a Gauss point pertaining to a FE close to the weak band of the specimen are also provided ${ }^{1}$. Finally, damage and equivalent strain distributions along a horizontal line (parallel to the loading direction) for five loading steps are depicted in Fig. 9 .

Numerical results evidence that the global response obtained using the ENL damage model is always in between the local and INL responses. Furthermore, the damage process evolution can be schematized according to the following phases:

(i) For null (or small) damage levels, ENL and INL damage models provide very similar global and local responses. In this phase the metric field is approximately uniform and equal to unity over the whole domain. As a consequence, effective geodesic distances do not strongly differ from Euclidean ones $\left(\tilde{\ell}_{x s}=\ell_{x s} \forall\left(\boldsymbol{x}_{x}, \boldsymbol{x}_{s}\right) \in \Omega\right)$. In other words, non-local interactions are still controlled by Euclidean distances between Gauss points, i.e., $\phi\left(\xi_{x s}^{\mathrm{ENL}}\right)=\phi\left(\xi_{x s}^{\mathrm{INL}}\right)$.

(ii) When damage increases, geodesic distances increase and become larger than Euclidean distances $\left(\tilde{\ell}_{x s}>\right.$ $\left.\ell_{x s}\right)$. Non-local interactions progressively reduce and the interaction zone shrinks, i.e., $\phi\left(\xi_{x s}^{\mathrm{ENL}}\right)<$ $\phi\left(\xi_{x s}^{\mathrm{INL}}\right)$. As a consequence, the ENL global response tends progressively to that obtained through the local evolution model.

(iii) Finally, when damage becomes approximately equal to unity on the weakest FE, the local and ENL damage responses become equivalent. In that case, geodesic interaction distances between Gauss integration points across the damaged zone tend to infinity and non-local interactions vanish. Such a response also ensures that no damage evolution occurs even through the sample elongation continues to increase. In this phase, the imposed external load mainly induces an increase in the horizontal

\footnotetext{
${ }^{1}$ Note that Geodesic interaction distances $\tilde{\ell}_{x s}>\ell_{c}$ are not depicted for sake of clarity. Accordingly, any point $\boldsymbol{x}_{s} \in \mathcal{H}$ pertaining to a blank region not more interacts with the reference Gauss point $\boldsymbol{x}_{x}$.
} 

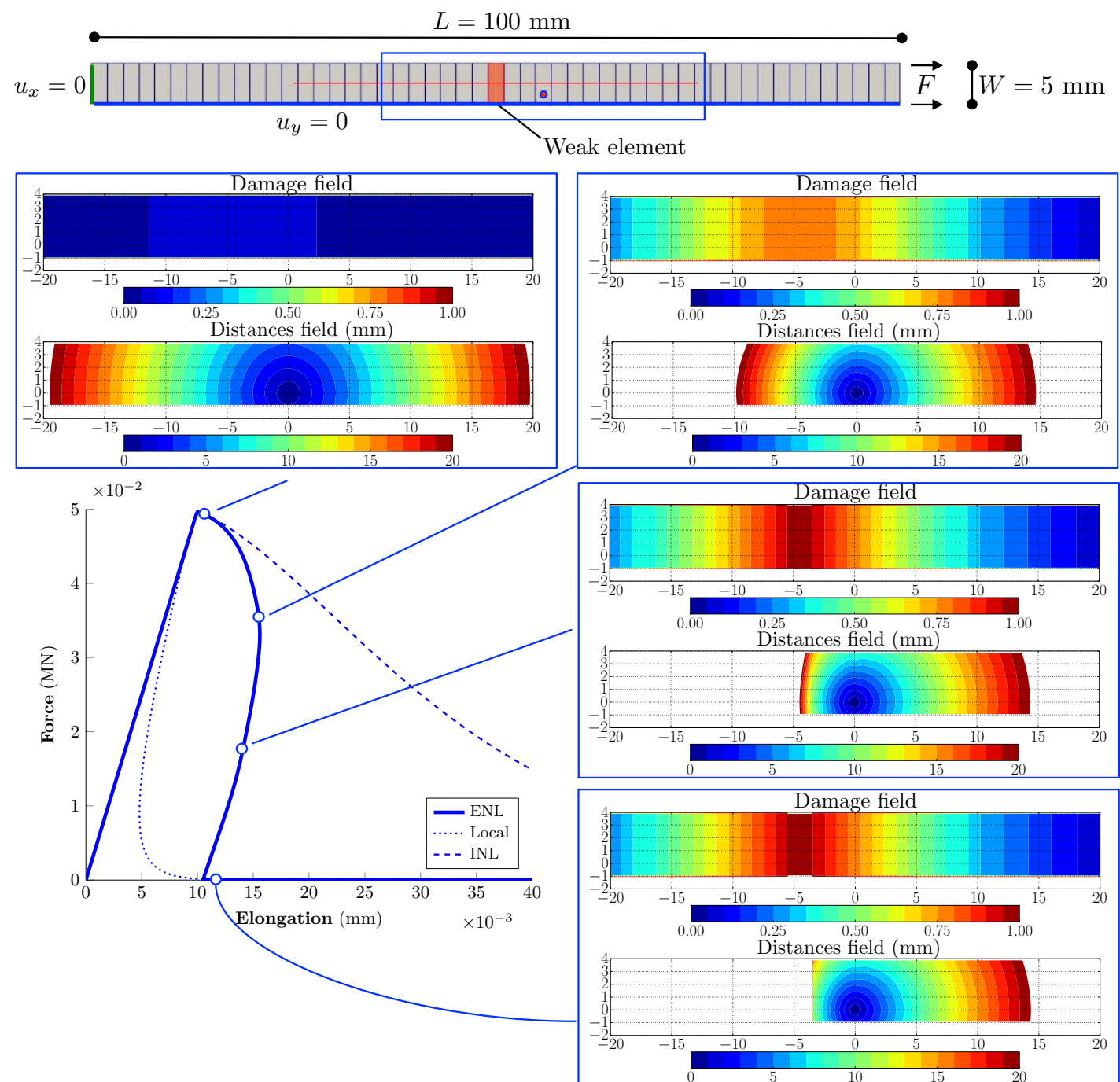

Figure 8: Tensile test. Comparison among representative global force - displacement responses obtained through the local, INL and ENL damage evolution models (FE mesh comprising 51 elements). Evolution of the non-local interaction distances $\left(\tilde{\ell}_{x s}\right)$ computed from a Gauss point pertaining to a FE located near the weak element for four test phases

strain field of the weakest element (as for the local damage model). As it is well known, this is not the case when considering the INL damage evolution model. In that case, Gauss points experiencing high damage levels still interact with their neighbors. Since for these integration points local equivalent strains attain very large values, non-local equivalent deformations computed on neighbor points continue increasing too. Damage evolution thus continues and the damaged band progressively widens. This spurious response is then avoided by using the ENL formulation.

\subsubsection{Fast Marching grid sensitivity}

Previous results where obtained by considering a FM grid refinement level $\rho=17$ for each Gauss point. According to criterion (23), this leads to use $601 \times 601=361^{\prime} 201$ vertexes for each Fm grid (for a FE mesh comprising 51 elements, $\ell_{x s, \min } \approx 0.83 \mathrm{~mm}$ ). As anticipated in previous sections, however, one could expect that parameter $\rho$ strongly influences the obtained response and should be chosen properly. To illustrate its role, a series of numerical computations was performed considering $\rho$ values ranging from 3 to 20 . Such a choice corresponds to using FM grids with a number of vertexes $N$ ranging between $101 \times 101=10^{\prime} 201$ and

Page 16 of 26 

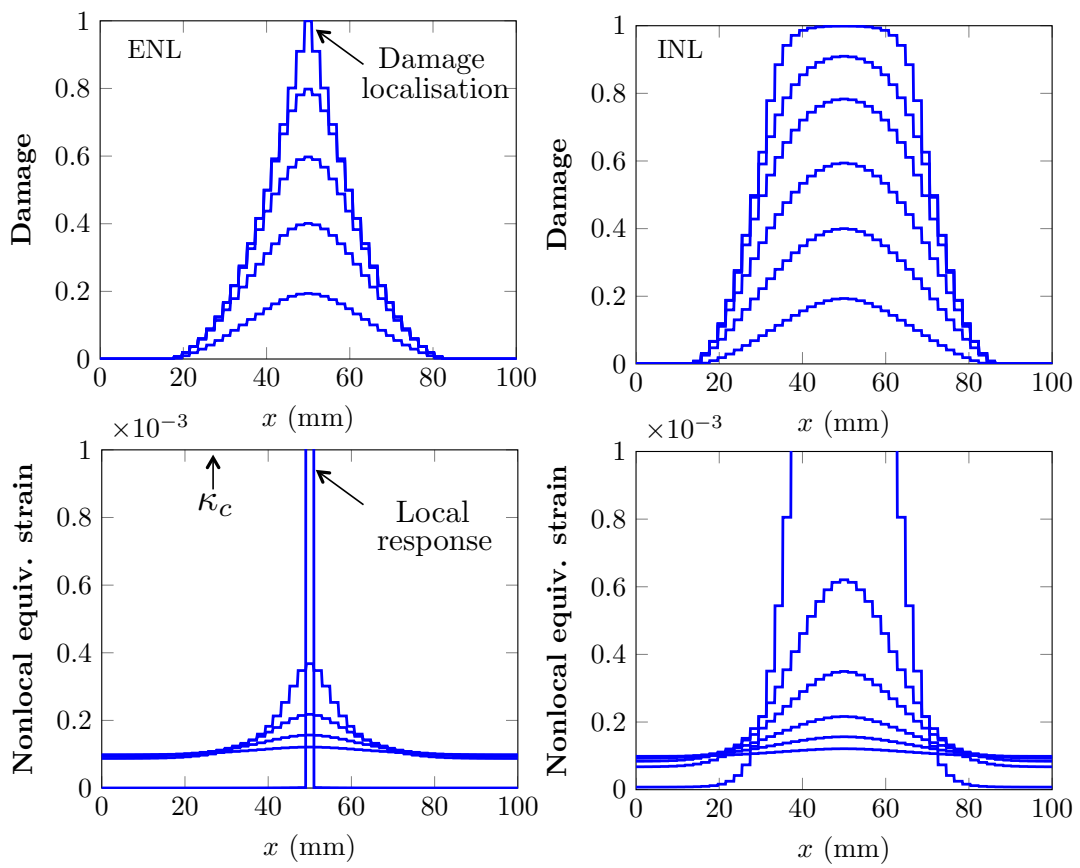

Figure 9: Tensile test. Damage and non-local equivalent strain fields for different phases of representative simulations carried out considering the ENL (left) and INL (right) damage evolution models

$801 \times 801=641^{\prime} 601$ (this latter is considered as the reference). According to results presented in Fig. 3 , the corresponding CPU times range from 0.0001s to 0.01s per FM computation (i.e., per Gauss point). As a consequence, for the considered case, the total CPU time associated to the computation of non-local interaction distances ranges from 0.02 to 2 seconds per loading step ( 51 elements $\times 4$ Gauss points / element per loading increment).

Numerical results provided in Fig. 10 evidence that using a small number of FM grid points can lead to a poor estimation of geodesic interaction distances, thus inducing an inaccurate mechanical response prediction. A progressive convergence towards the reference solution can be however observed for $\rho \geq 8$. This suggests that a less restrictive criterion for defining $h^{\mathrm{FM} \text {,max }}$ could be used without strongly influencing the obtained response. Note however, that the considered case is quite special, because each pair of nearest Gauss points is aligned horizontally. Furthermore, due to the FE's elongation along the $y$ axis direction, vertically/horizontally unaligned points are always far enough to be computed properly. Further computations are therefore needed in order to better define an objective criterion for properly choosing the FM grid spacing, thus optimizing computational resources. This criterion will strictly depend on the adopted FM algorithm (first/second order, triangular/quadrangular grids, ...) as well as on the algorithmic strategy chosen for computing distances between Gauss points from non-matching FM grids.

\subsubsection{FE mesh sensitivity}

The objectivity of the obtained solution with respect to the FE discretization can be verified by comparing global response obtained for three structured mesh with characteristic FEs' sizes $\left(h^{\mathrm{FE}}\right)$ ranging between $L / 101=0.99 \mathrm{~mm} \approx \ell_{c} / 20$ and $L / 27=3.70 \mathrm{~mm} \approx \ell_{c} / 5.5$. This ensures that non-local horizons $\mathcal{H}$ of each Gauss point contain a number enough of integration points for properly computing non-local averaged quantities.

A good agreement among obtained force-displacement responses is observed in the whole range of explored displacements (Fig. 11). A tendency toward a mesh convergence is clear and corroborates the regularization properties of the proposed formulation (similar considerations hold when considering different sets of materials parameters). Damage profiles obtained for the three FE meshes are also extremely 


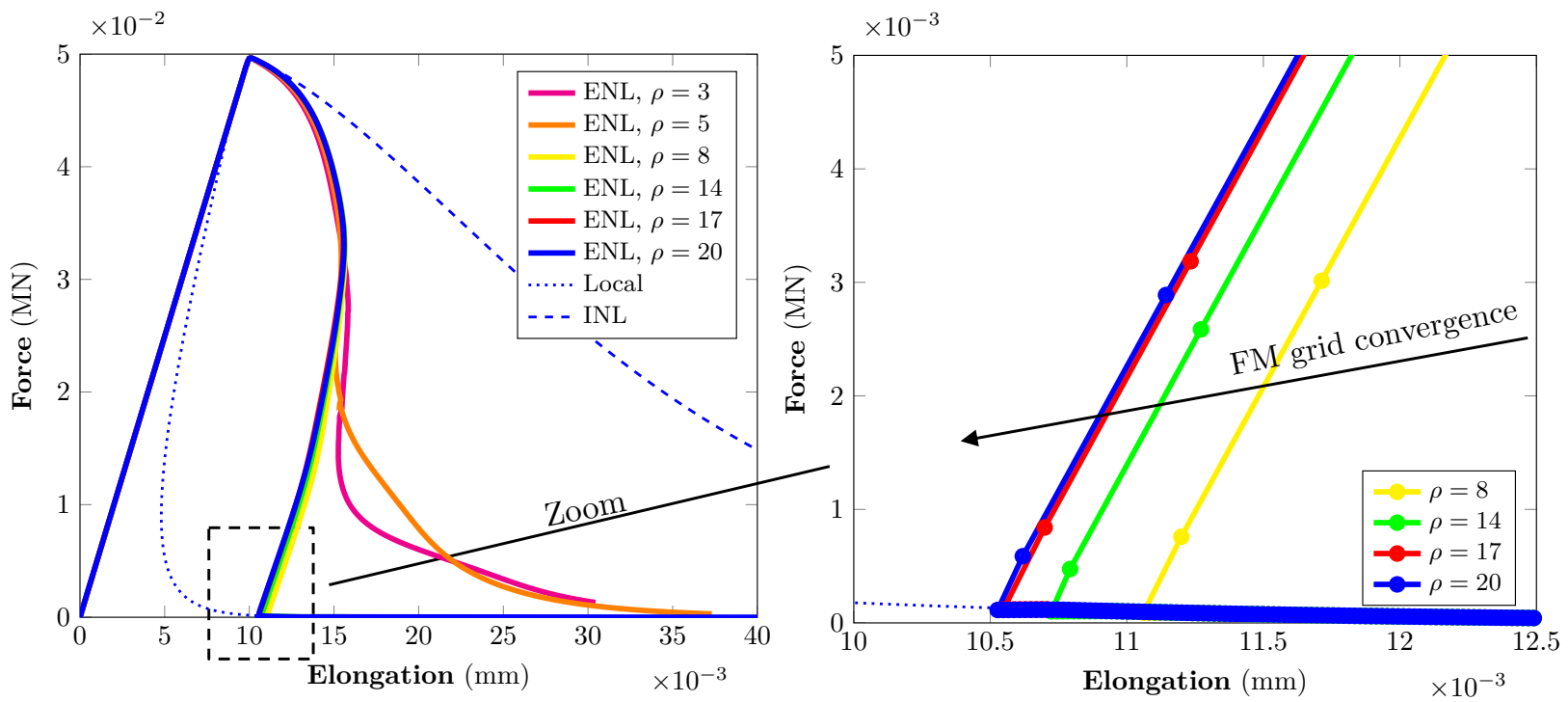

Figure 10: Tensile test. FM mesh sensitivity: global force - displacement responses obtained considering different FM grid refinement levels with parameter $\rho$ ranging from 3 to 20 (FE mesh comprising 51 elements)

similar for any damage level.

Very small differences in global responses can be observed only on final simulation phases (i.e., close to total failure of the bar), when high damage levels on the central/weak element induce non-local interactions to vanish. This response is common to other non-local models with evolving internal length (e.g. Giry et al. (2011)) and should not be interpreted as representative of a mesh dependency issue. Indeed, the separation of the global curves corresponds to the onset of the transition toward a purely local response of the central element. As this transition occurs for very high damage levels ( $d$ larger than approximately 0.95 ), the "residual" energy to be dissipated in a local manner on the weakest element is very small if compared to the energy dissipated throughout the whole damaging process.

Strategies to further improving mesh insensitivity. In order to remove also these small differences, different strategies can be explored:

(i) A simple choice could consists in modifying the formulation by stopping updating the Riemannian metric field when the damage level exceeds a given threshold value. From a numerical viewpoint, this can be obtained by defining the Riemann metric field as: $m_{n}(\boldsymbol{x})=\sqrt{\left.1-\min \left(d_{n}(\boldsymbol{x}), d_{\max }^{\mathrm{FM}}\right)\right)}>0$. As it will be shown in the following, however, this approach has a strong drawback: interactions between the weakest elements ant its neighbors do not vanish, leading to damage diffusion across the damage band (as in standard INL formulations). In order to illustrate this situation, global responses obtained for three threshold damage values $d_{\max }^{\mathrm{FM}}(0.99,0.95$ and 0.9$)$ are compared in Fig. 12a. When the threshold value is small enough $(<0.95)$, the mesh insensitivity is recovered in the whole range of explored displacements. As a counterpart of this, however, assuming a non vanishing metric field induces residual interactions between Gauss points to exist even through the central element is completely damaged. As a consequence, damage diffusion across the strain localization area (for high elongation levels) cannot be avoided a-priori. As an example of this, Fig. 12b depicts damage and non-local equivalent strains evolution for $d_{\max }^{\mathrm{FM}}=0.9$ (for a $\mathrm{FE}$ mesh comprising 51 elements). This clearly shows that once the condition $d \rightarrow 1^{-}$is attained on the weaker element, non-local strains remains non null over a damaged band finite in width and damage is progressively spread. Provided that these issues are completely absent in the standard ENL formulation (Sec. 2), one can conclude that thresholding the metric field is an ineffective strategy. 

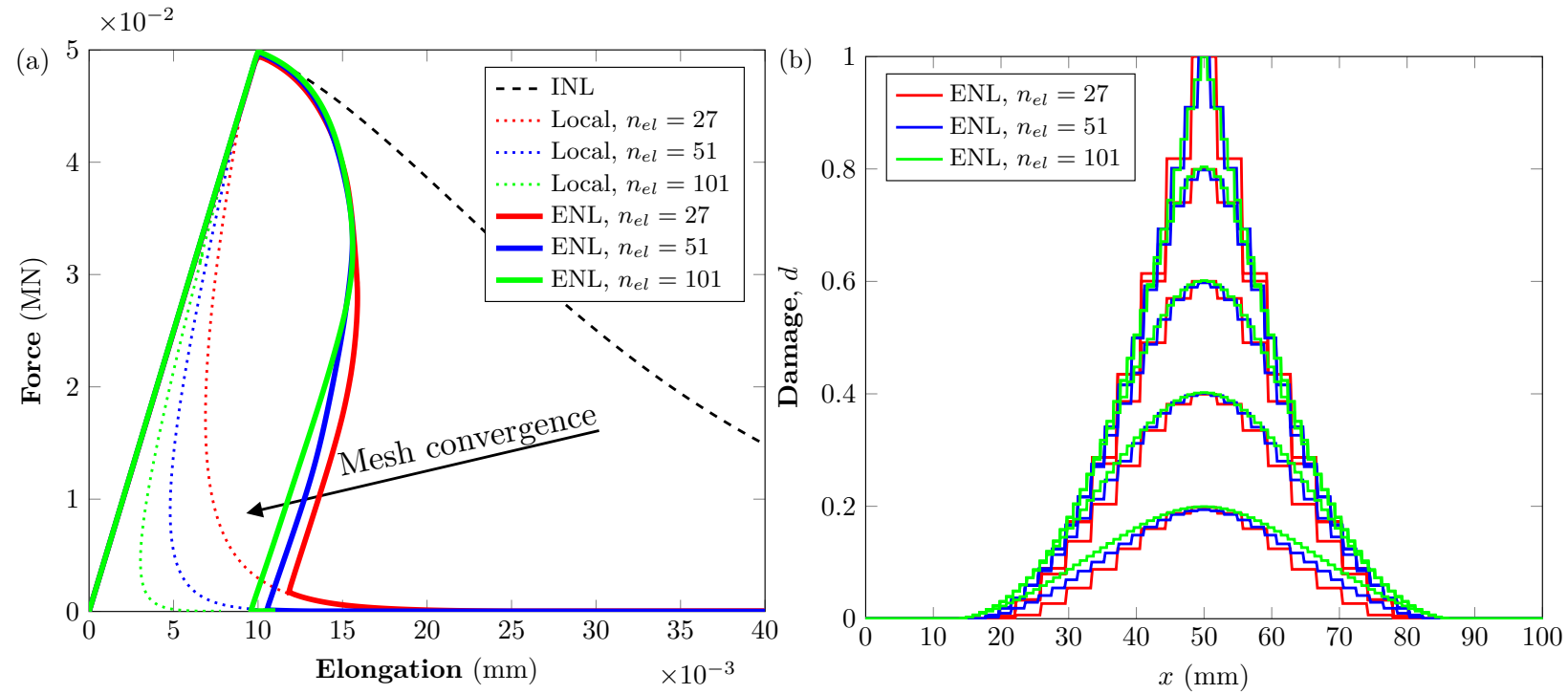

Figure 11: Tensile test. FE mesh sensitivity: global force - displacement responses and damage distributions obtained for three FE mesh refinement levels $\left(n_{e l}=27,51\right.$ and 101 elements)

(ii) A second possible strategy could consist in enhancing the numerical formulation in order to model a continuous-to-discontinuous kinematics transition once a given threshold damage value is reached. Indeed, when the width of the localization band tends to a value smaller than the element size, the displacement field can no more be well described in a standard FEM framework. Several formulations providing consistent thermodynamic frameworks for evolving from non-local damage to strong discontinuity models were proposed in the literature (e.g. Jirásek and Zimmermann (2001), Simone et al. (2003), Cazes et al. (2009), Tamayo-Mas and Rodríguez-Ferran (2014)). Such a transition could ensure to dissipate the residual energy no more on a single element (as in local models) but at the strong discontinuity level through a "cohesive zone model" (formulated in the Embedded FEM, eXtended FEM, ...). Although interesting, such a formulation would induce an increased numerical complexity and is left for further works.

\subsection{Wedge splitting test}

The wedge splitting test is a classic fracture mechanics experimental test (Brühwiler and Wittman, 1990) used to estimate the fracture energy of concrete and concrete-like materials (e.g. concrete, mortars). Here, it is simulated in order to provide some information concerning the key features of the damage propagation process when using a ENL damage model.

The computational domain represents a vertically notched specimen $200 \mathrm{~mm}$ in width and $200 \mathrm{~mm}$ in height (Fig. 13). It is discretized through a FE mesh comprising $n_{e l}=2690$ quadrilateral FEs with linear interpolation of the displacement field. The elementary characteristic size in the central part of the specimen is $h^{\mathrm{FE}}=3 \mathrm{~mm}$. A sub-vertical crack propagation, from the notch $(3 \mathrm{~mm}$ in width $)$ to the bottom of the sample, is induced by directly imposing monotonic increasing horizontal displacements of the bearing surfaces. ENL computations are performed considering very refined FM grids $(\rho=17)$ as suggested in previous sections. Furthermore, damage evolution is supposed as non-local only in the central part of the specimen, where high stress-strain gradients are expected and damage will occur. Material parameters are assigned as follows: $E=100 \mathrm{MPa}, \nu=0.2, \kappa_{0}=0.0001, \kappa_{c}=5 \times \kappa_{0}=0.0005$, while the characteristic/internal length is assumed equal to $\ell_{c}=5 \times h^{\mathrm{FE}}=15 \mathrm{~mm}$. 

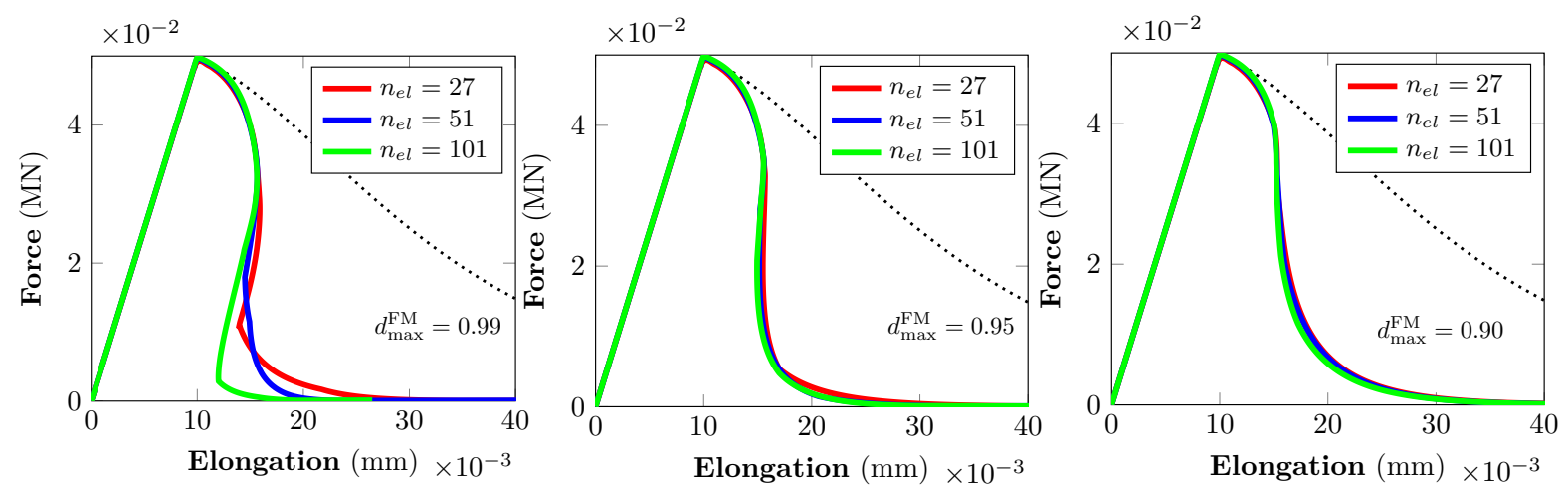

(a)
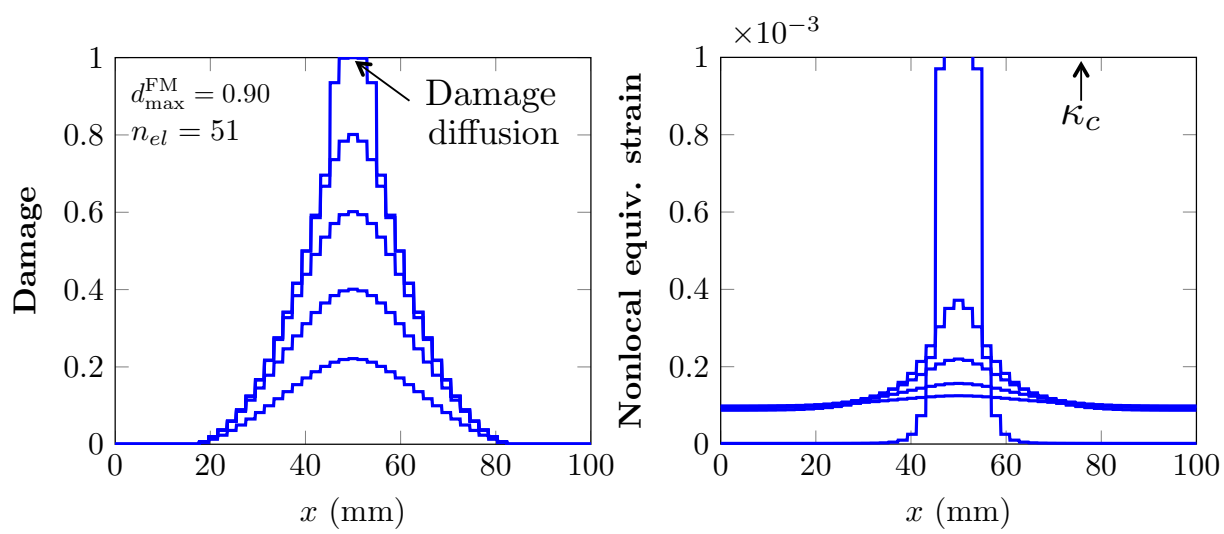

Figure 12: Tensile test. Ineffective thresholding of the metric field to improve FE mesh sensitivity: a) global responses obtained for three FE mesh refinement levels using a ENL formulation with a bounded damage dependent Riemannian metric field (three threshold damage levels: $0.95,0.9,0.99)$; b) Damage and non-local equivalent strain fields for different phases of representative simulations carried out considering a ENL formulation with a bounded damage dependent Riemannian metric field $\left(d_{\max }^{\mathrm{FM}}=0.9\right.$, $\left.n_{e l}=51\right)$

\subsubsection{ENL and INL damage models}

The global response of the sample is represented through the total reaction force on the bearing surfaces and their relative displacement (imposed). Alternatively one can also use the crack opening displacement (COD) at the notch level (as defined in Fig. 13). Figure 14 compares structural responses obtained through the INL and ENL formulations. Damage distributions for three imposed displacement levels are also compared in Fig. 15. As expected, one observes that:

(i) Global responses and damage evolution processes are quite similar in the early phases of the test, until the damage level in front of the notch (at the location where the non-local equivalent strain first satisfies the damage activation criterion) remains small. In this phase, geodesic and Euclidean distances almost coincide.

(ii) Once this material point starts experiencing significant damage levels $(d>0.1$ approximately), geodesic distances start increasing and non-local interactions within its neighborhood decrease (for the ENL formulation) thus leading to a progressive damage localization. As a consequence of strain localization, the maximum load predicted by the ENL formulation is lower than that provided by the INL model.

(iii) For more advanced loading stations (imposed relative external displacements), the damage field predicted using the INL formulation is diffuse over a large zone across the symmetry axis, i.e., $d(\boldsymbol{x})$ is approximately unity over a large damaged band. Conversely, the ENL formulation provides a better description of the damaging process. In that case, the fully damaged condition is attained over the 

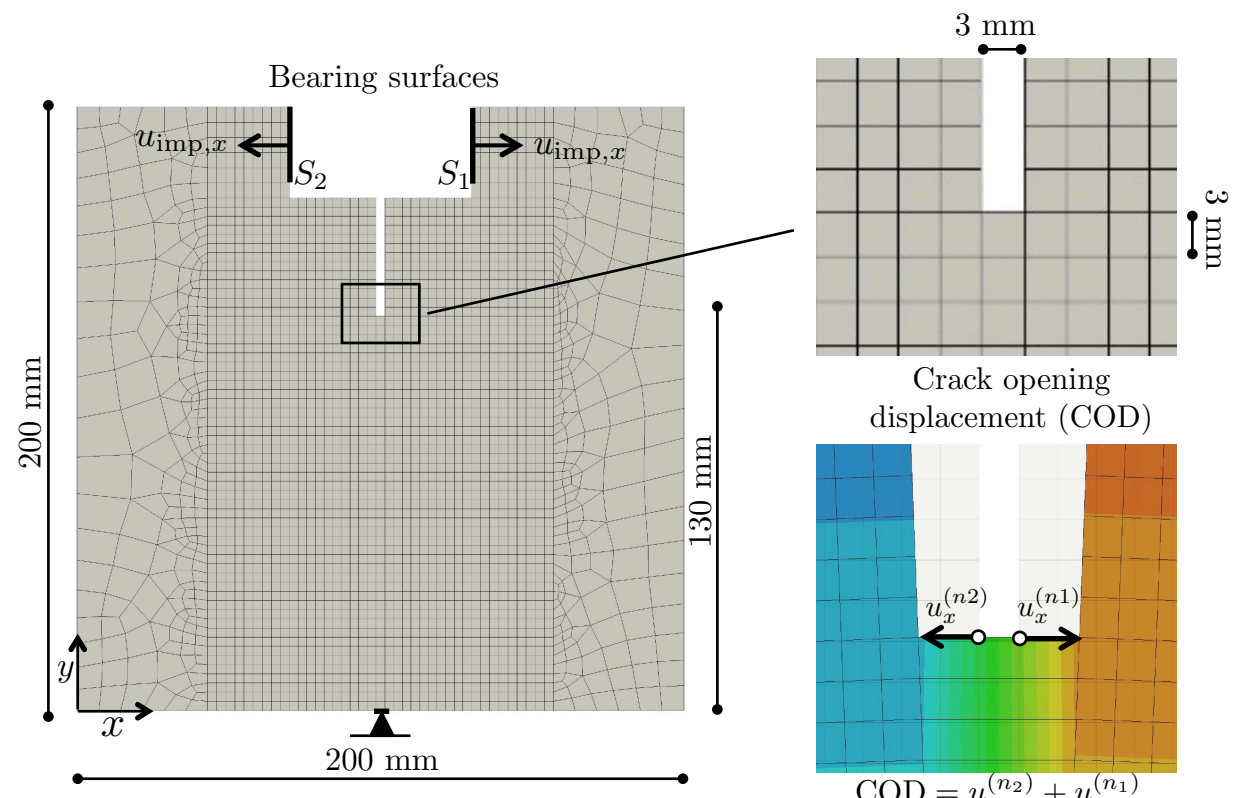

Crack opening displacement (COD)

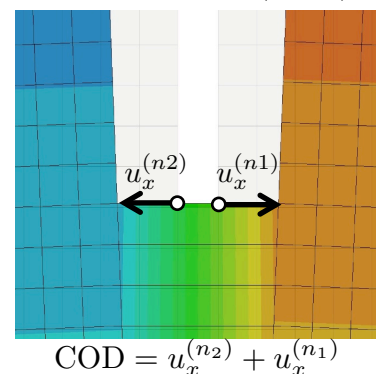

Figure 13: Wedge splitting test. Sample geometry, boundary conditions and finite element discretization

central line of elements (aligned with the damage propagation direction) as classically predicted by local models and experimentally observed with a unique crack localized. Furthermore, damage decreases rapidly to null values when moving away from it. Despite what is observed using INL formulations, once the condition $d \approx 1$ is attained on the symmetry axis, equivalent strains continue increasing at this location. However, as with ENL formulations non-local geodesic interaction distances tends to infinity, the damage field no more evolves. By this way, the damage diffusion across the damaged band and in the backward of the notch is strongly reduced even through very high deformation levels are attained on the center of the damaged band. Note that, for the last damage distribution of Fig. 14, the horizontal strain level registered in correspondence of the notch is approximately equal to $0.09 \approx 250 \times \kappa_{0}$ and $50 \times \kappa_{c}$.

\subsubsection{Damage propagation process}

It is well known (Bazant, 1986) that cracking/damaging processes in quasi-brittle materials are characterized by the formation and growth of distributed micro-cracks in front of the crack tip first. This region is characterized by a notable dissipated energy and induces a nonlinear material response. Then, some microcracks progressively coalesce into a single dominant macro-crack when loading increases. In this phase, strain concentration in a band of small thickness occurs, micro-cracks surrounding the macro-crack progressively arrest and a stress relaxation in the neighborhood of the macro-crack occurs. Once this condition is attained two novel stress-free surfaces are formed.

Better studying the wedge-splitting test simulation allows for showing that the ENL formulation naturally represents these phases, at least in qualitative terms. For this purpose, damage and non-local equivalent strain fields corresponding to the loading step (2) of Fig. 14 are provided in Fig. 16. One observes that:

(i) the damaged band, approximately $2 \ell_{c}$ in thickness, is always preceded by a zone of finite width where dissipation occurs (i.e., the non-local yield criterion is satisfied and damage evolves). Material points pertaining to this zone interact between them till non-local interaction distances are lower than the internal characteristic length. Furthermore, higher is the damage level at the considered material point lower non-local interactions (i.e., a tendency toward a local response is modeled). 

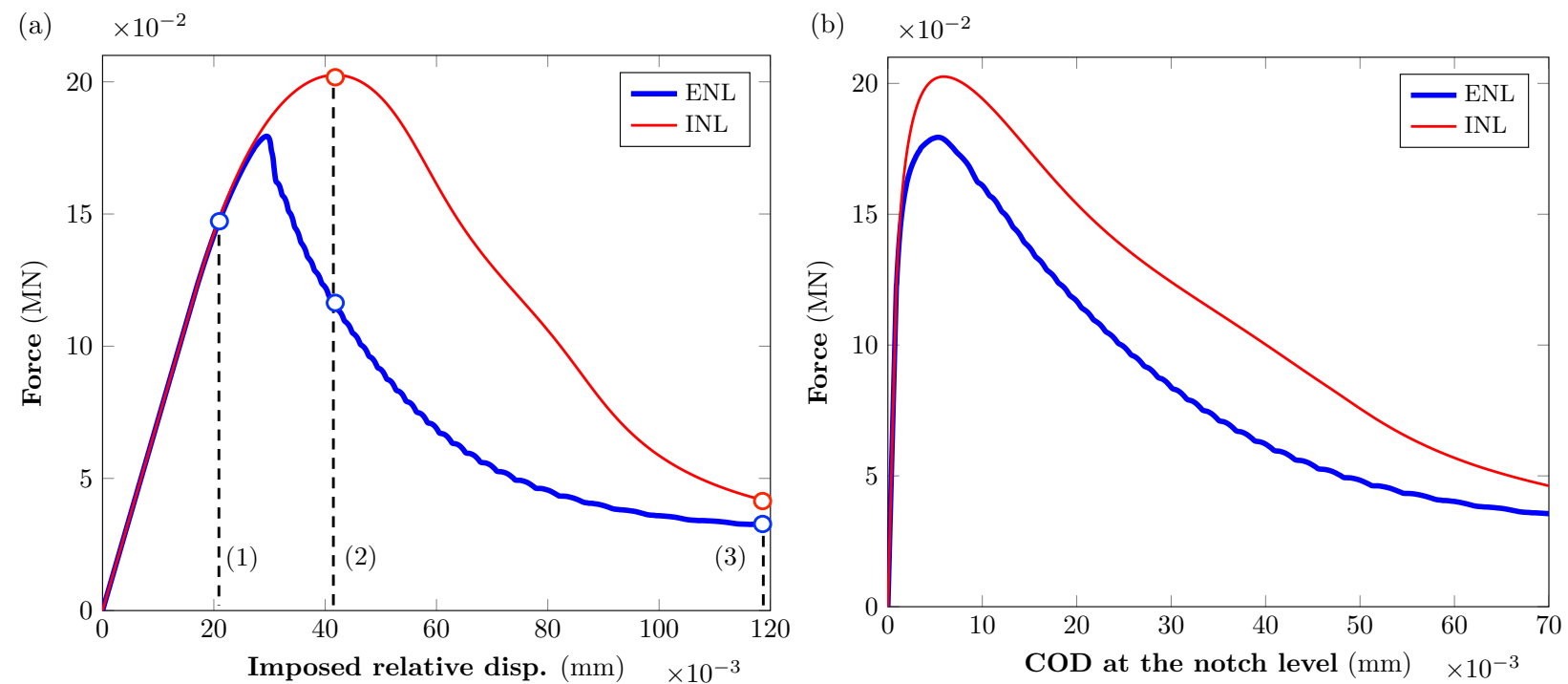

Figure 14: Wedge splitting test. Global sample responses obtained through the ENL and INL formulations: a) force vs relative displacement of the bearing surfaces (with identification of the displacement levels corresponding to the damage fields depicted in Fig. 15); b) force vs Crack Opening Displacement (COD) at the notch level

(ii) In the proposed model, strain localization corresponds to the phase when damage tends to unity and strains localize on the axis of the damaged band (considered as representative of a "macro-crack"). Once this condition is achieved, damage evolution at material points located close to the "marco-crack" progressively arrest (non-local interactions vanish). These material points continue experiencing slight damage evolutions till the damaged band advances enough to remove all interactions with material points located on the damage propagation front and on the other side of the damaged band.

(iii) Finally, provided that damage on the center of the damaged band is very close to unity, a stress release in the surrounding material occurs. As a consequence, further increases in the external actions result in a strain increase at the most damaged material points only (non-local equivalent strains tend to infinity on the central line of elements only), thus leading to a progressive "macro-crack" opening.

\section{Conclusive remarks}

A numerical formulation for modeling damage dependent non-local interactions through a Eikonal NonLocal (ENL) Continuum Damage model was presented in this paper. The ENL formulation (Desmorat et al., 2015) provides a novel interpretation of damage dependent evolving non-local interactions. From a differential geometry viewpoint, this leads to consider that damage induces a curvature of the Riemannian space in which interaction distances are computed. From a mathematical point of view, interaction distances are computed as solutions of an isotropic time-independent Eikonal equation with a damage dependent metric function. The numerical implementation was performed by coupling Fast-Marching (Sethian, 1996) algorithms, for computing interaction distances, and a standard Finite Element (FE) procedure, for solving the quasi-static equilibrium equations. The Mazars isotropic damage model (Mazars, 1984) with a single scalar damage variable was considered in this work. The proposed formulation is however completely general, and can be extended to any non-local damage model without major modifications.

Quasi-static localization examples showed the ability to perform FE computations within a ENL framework. A tensile test was modeled to illustrate the influence of damage on evolving non-local interactions first. This allowed showing that the global response obtained through the ENL formulation follows that obtained through the INL model (Pijaudier and Bazant, 1987) for small damage levels, while tends to the local 

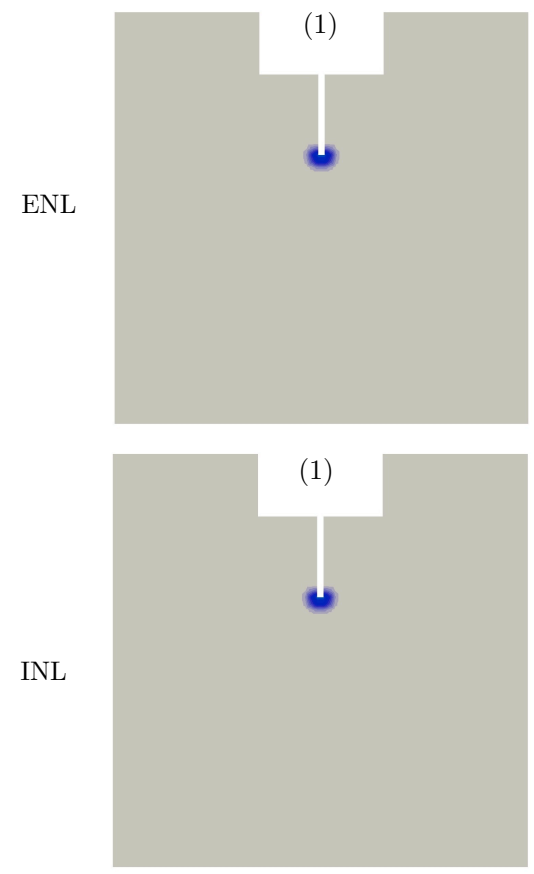

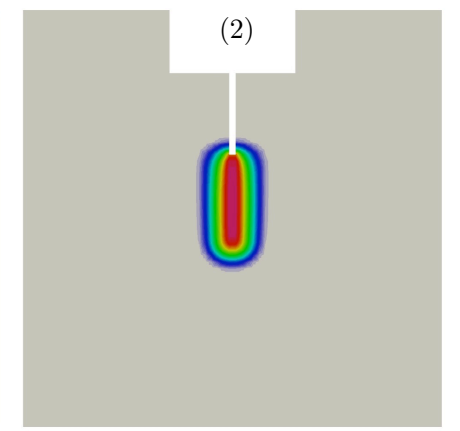

$(2)$

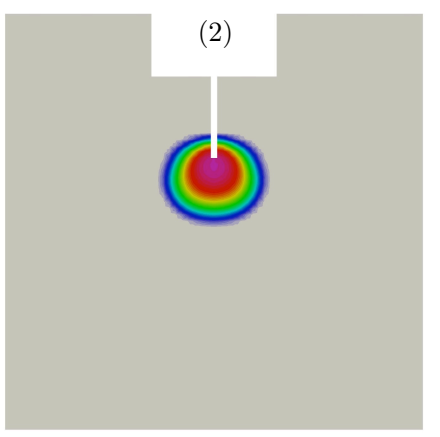

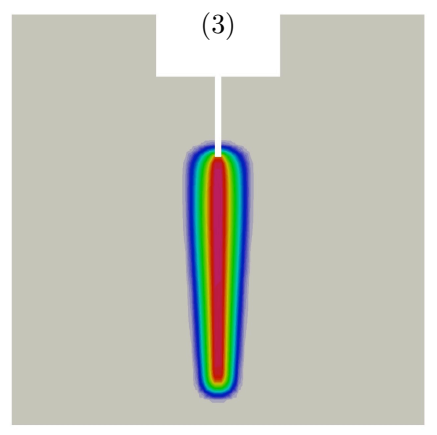
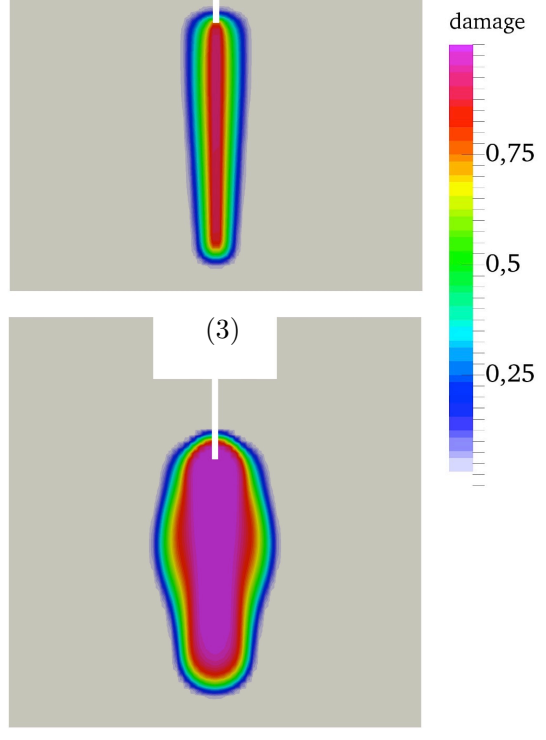

Figure 15: Wedge splitting test. Comparison among damage fields computed by considering INL and ENL damage evolution models for three loading stations (the corresponding displacement levels are defined in Fig. 14)

one when damage grows up to unity. Furthermore, when the damaged band is completely developed, the ENL framework ensures the absence of some pathologic damage diffusion problems, as classically associated with standard INL formulations. According to the ENL formulation, in fact, material points located across the damaged band no longer interacts. In this phase, any displacement increment imposed to the system induces a localized strain increase without any damage evolution, as in local damage model. Despite what is classically observed with local damage evolution models, the mesh dependency of the global response is however strongly reduced. The ENL formulation thus provides a good alternative to standard INL methods for regularizing the localization problem when the material experiences some softening. The simulation of a wedge splitting test (Brühwiler and Wittman, 1990) further confirmed these observations. Furthermore, this allowed showing that during the strain localization process, the damaged front is always preceded by a region finite in size where the damage evolution still remains non-local. In other words, a sort of fracture/damage process zone is naturally represented.

Further works are needed in order to solve numerically more complex mechanical problems and to better understand the regularization properties of the ENL formulation (from a theoretical viewpoint). Some systematic study concerning the robustness of such an implementation would also be interesting. In particular for what concerns the chosen FM algorithm and the optimal value of the FM grid spacing to be used for effective distances computation. The extension to an anisotropic damage context could also represent an important perspective. In this latter case, the main enhancement required will be numerical and concern the adoption of an anisotropic Fast-Marching algorithm (Peyré et al., 2010) (the Riemannian metric function becomes tensorial and anisotropic).

A comparison with a stress-based non-local formulation (Giry et al., 2011) could also be useful. Note since now, however, that a key differences between these methods consists in the fact that in ENL formulations interactions between two material points are influenced by the whole damage field between them, whereas in the stress-based formulation interactions are driven by the stress state of the sole "emitting" point $\boldsymbol{x}_{x}$. As a consequence of this, two points separated by an highly damaged zone reduce their interaction through the reduction in the stress level associated with the damaging process. The main theoretical, numerical and 


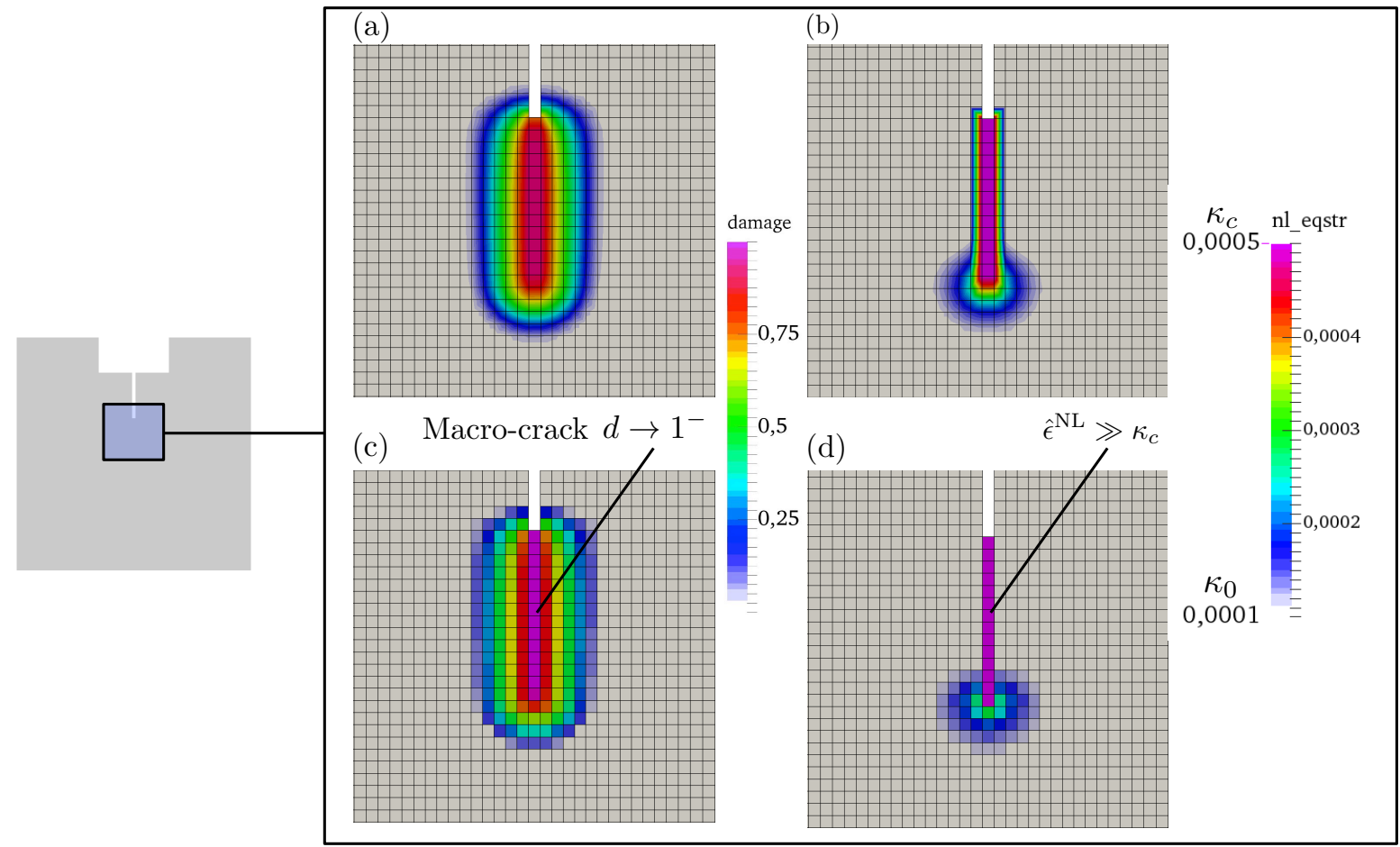

Figure 16: Wedge splitting test. Damage and non-local equivalent strain fields for the same loading step (nodal projections $(\mathrm{a}-\mathrm{b})$ and mean values over elements (c-d))

algorithmic features of these formulations should be therefore compared deeply.

Finally, provided the capabilities of the ENL model in representing strain localization, its coupling with strong discontinuity methods (e.g. Embedded FEM, eXtended FEM, ... ) could be explored. Introducing a strong discontinuity once damage localizes over a single line of elements might further improve regularization features of the ENL formulation when non-local interactions vanish. Furthermore, one could obtain explicit information concerning crack opening displacements and orientations. This could be particularly useful in more complex cese studies involving curved and/or branching cracks. By this way, a large series of engineering problems (e.g. fluid leakage rates estimation in containment structures (Pijaudier-Cabot et al., 2009, Rastiello et al., 2014, 2015, 2016), coupled cracking-diffusion phenomena in quasi-brittle materials (Secchi and Schrefler, 2012, Heider and Markert, 2017)) could be modeled directly.

\section{Acknowledgements}

All authors listed have contributed to the research project to be included as authors. The authors declare no potential conflicts of interest with respect to the research, authorship, and/or publication of this article. The authors received no financial support for the research, authorship, and/or publication of this article.

\section{References}

Z. P. Bazant. Mechanics of distributed cracking. Appl. Mech. Rev, 39(5):675-705, 1986.

Z. P. Bažant and M. Jirásek. Nonlocal integral formulations of plasticity and damage: survey of progress. Journal of Engineering Mechanics, 128(11):1119-1149, 2002.

Z. P. Bažant and M. Jirásek. Nonlocal Integral Formulations of Plasticity and Damage: Survey of Progress. Journal of Engineering Mechanics, 128(11):1119-1149, 2002. ISSN 0733-9399. doi: 10.1061/(ASCE)0733-9399(2002)128:11(1119). 
Z. P. Bazant, J.-L. Le, and C. G. Hoover. Nonlocal boundary layer (nbl) model: overcoming boundary condition problems in strength statistics and fracture analysis of quasibrittle materials. Fracture Mechanics of Concrete and Concrete Structures-Recent Advances in Fracture Mechanics of Concrete, B.-H. Oh, Ed., Korea Concrete Institute, Seoul, pages 135-143, 2010.

D. P. Bertsekas. A simple and fast label correcting algorithm for shortest paths. Networks, 23(8):703-709, 1993.

E. Brühwiler and F. H. Wittman. The Wedge splitting test, a new method of performing stable fracture mechanics tests. Enginnering Fracture Mechanics, 35:117-125, 1990.

F. Cazes, M. Coret, A. Combescure, and A. Gravouil. A thermodynamic method for the construction of a cohesive law from a nonlocal damage model. International Journal of Solids and Structures, 46(6):1476 - 1490, 2009. ISSN 0020-7683. doi: https: //doi.org/10.1016/j.ijsolstr.2008.11.019. URL http://www.sciencedirect.com/science/article/pii/S0020768308004903.

J. Cordebois and F. Sidoroff. Endommagement anisotrope en élasticité et plasticité. JMTA, Numéro spécial, pages 45-60, 1982.

T. H. Cormen, C. E. Leiserson, R. L. Rivest, and C. Stein. Introduction to algorithms, volume 6. MIT press Cambridge, 2001.

R. de Borst, M. A. Crisfield, J. J. Remmers, and C. Verhoosel. Nonlinear Finite Element Analysis of Solids and Structures, 2nd Edition, 2012. URL http://eu.wiley.com/WileyCDA/WileyTitle/productCd-0470666447.html\{\#\}.

J. de Vree, W. Brekelmans, and M. van Gils. Comparison of nonlocal approaches in continuum damage mechanics. Computers \& Structures, 55(4):581-588, may 1995. ISSN 00457949. doi: 10.1016/0045-7949(94)00501-S. URL http: //www.sciencedirect.com/science/article/pii/004579499400501S.

R. Desmorat. Anisotropic damage modeling of concrete materials. International Journal of Damage Mechanics, 25(6):818-852, 2016.

R. Desmorat and F. Gatuingt. Introduction of an internal time in nonlocal integral theories. Technical report, LMT-Cachan, Cachan, France, 2007. URL http://dx.doi.org/10.1201/b10546-15.

R. Desmorat, F. Gatuingt, and F. Ragueneau. Nonlocal anisotropic damage model and related computational aspects for quasi-brittle materials. Engineering Fracture Mechanics, 74(10):1539-1560, 2007.

R. Desmorat, F. Gatuingt, and M. Jirásek. Nonlocal models with damage-dependent interactions motivated by internal time. Engineering Fracture Mechanics, 142:255-275, jul 2015. ISSN 00137944. doi: 10.1016/j.engfracmech.2015.06.015. URL http://www.sciencedirect.com/science/article/pii/S0013794415002787.

E. W. Dijkstra. A note on two problems in connexion with graphs. Numerische mathematik, 1(1):269-271, 1959.

S. Fichant, C. La Borderie, and G. Pijaudier-Cabot. Isotropic and anisotropic descriptions of damage in concrete structures. Mechanics of Cohesive-frictional Materials, 4(4):339-359, 1999.

M. Frémond and B. Nedjar. Damage, gradient of damage and principle of virtual power. International Journal of Solids and Structures, 33(8):1083-1103, 1996.

M. Geers, R. De Borst, W. Brekelmans, and R. Peerlings. Strain-based transient-gradient damage model for failure analyses. Computer methods in applied mechanics and engineering, 160(1-2):133-153, 1998.

C. Giry, F. Dufour, and J. Mazars. Stress-based nonlocal damage model. International Journal of Solids and Structures, 48 (25):3431-3443, 2011.

B. C. Hall. Quantum Theory for Mathematicians. 2013. ISBN 978-1-4614-7115-8. doi: 10.1007/978-1-4614-7116-5. URL http://www. springer.com/new $+\{\%\} 26+$ forthcoming+titles $+\{\%\} 28$ def ault $\{\%\} 29 /$ book/978-1-4614-7115-8 $\$ \backslash$ delimiter"026E30F\$npapers2://publication/doi/10.1007/978-1-4614-7116-5.

D. Halm and A. Dragon. An anisotropic model of damage and frictional sliding for brittle materials. European Journal of Mechanics-A/Solids, 17(3):439-460, 1998.

Y. Heider and B. Markert. A phase-field modeling approach of hydraulic fracture in saturated porous media. Mechanics Research Communications, 80(Supplement C):38 - 46, 2017. ISSN 0093-6413. doi: https://doi.org/10.1016/j.mechrescom.2016.07.002. URL http://www.sciencedirect.com/science/article/pii/S0093641316300714. Multi-Physics of Solids at Fracture.

T. Hughes. The Finite Element Method: Linear Static and Dynamic Finite Element Analysis. Dover Civil and Mechanical Engineering. Dover Publications, 2000. ISBN 9780486411811. URL https://books.google.ie/books?id=yarmSc7ULRsC.

M. Jirásek and T. Zimmermann. Embedded crack model. part ii: combination with smeared cracks. International Journal for Numerical Methods in Engineering, 50(6):1291-1305, 2001. ISSN 1097-0207. doi: 10.1002/1097-0207(20010228)50:6<1291:: AID-NME12>3.0.CO;2-Q. URL http://dx.doi.org/10.1002/1097-0207(20010228) 50:6<1291: :AID-NME12>3.0.C0;2-Q.

R. Kimmel and J. A. Sethian. Computing geodesic paths on manifolds. Proceedings of the National Academy of Sciences, 95 (15):8431-8435, 1998.

A. Krayani, G. Pijaudier-Cabot, and F. Dufour. Boundary effect on weight function in nonlocal damage model. Engineering Fracture Mechanics, 76(14):2217-2231, 2009.

J. Lemaitre and R. Desmorat. Continuum Damage Mechanics. Springer Berlin Heidelberg, 2005. ISBN 978-3-540-21503-5. doi: 10.1007/3-540-27293-3\{\_\}1. URL http://dx.doi.org/10.1007/3-540-27293-3\{_\}1.

J. Marigo. Formulation d'une loi d'endommagement d'un matériau élastique. CR Acad. Sci. Paris II, 292:1309-1312, 1981.

J. Mazars. Application de la mécanique de l'endommagement au comportement non linéaire et à la rupture du béton de structure. PhD thesis, 1984.

C. Miehe, F. Welschinger, and M. Hofacker. Thermodynamically consistent phase-field models of fracture: Variational principles and multi-field FE implementations. International Journal for Numerical Methods in Engineering, 83(10):1273-1311, sep 2010. ISSN 00295981. doi: 10.1002/nme.2861. URL http://doi.wiley.com/10.1002/nme.2861.

N. Moës, C. Stolz, P.-E. Bernard, and N. Chevaugeon. A level set based model for damage growth: The thick level set approach. International Journal for Numerical Methods in Engineering, 86(3):358-380, apr 2011. ISSN 00295981. doi: 10.1002/nme.3069. URL http://doi.wiley.com/10.1002/nme.3069.

N. Moës, C. Stolz, and N. Chevaugeon. Coupling local and non-local damage evolutions with the Thick Level Set 
model. Advanced Modeling and Simulation in Engineering Sciences, 1(1):16, oct 2014. ISSN 2213-7467. doi: 10.1186/ s40323-014-0016-2. URL http://www. amses-journal.com/content/1/1/16.

S. Murakami and N. Ohno. A constitutive equation of creep damage in pollicristalline metals. In IUTAM Colloquium Euromech, volume 111, 1978

G. D. Nguyen. A damage model with evolving nonlocal interactions. International Journal of Solids and Structures, 48(10): $1544-1559,2011$.

S. A. Orszag and C. Bender. Advanced mathematical methods for scientists and engineers. Mac Graw Hill, 1978.

R. Peerlings, M. Geers, R. de Borst, and W. Brekelmans. A critical comparison of nonlocal and gradient-enhanced softening continua. International Journal of Solids and Structures, 38(44-45):7723-7746, nov 2001. ISSN 00207683. doi: 10.1016/ S0020-7683(01)00087-7. URL http://www.sciencedirect.com/science/article/pii/S0020768301000877.

G. Peyré, M. Péchaud, R. Keriven, L. D. Cohen, et al. Geodesic methods in computer vision and graphics. Foundations and Trends@ in Computer Graphics and Vision, 5(3-4):197-397, 2010.

C. G. Pijaudier and Z. P. Bazant. Non local Damage Theory. Asce, 113(10):1512-1533, 1987. doi: http://dx.doi.org/10.1061/ (ASCE)0733-9399(1987)113:10(1512).

G. Pijaudier-Cabot and A. Benallal. Strain localization and bifurcation in a nonlocal continuum. International Journal of Solids and Structures, 30(13):1761-1775, 1993.

G. Pijaudier-Cabot and F. Dufour. Non local damage model: boundary and evolving boundary effects. European Journal of Environmental and Civil Engineering, 14(6-7):729-749, 2010.

G. Pijaudier-Cabot, K. Haidar, and J.-F. Dubé. Non-local damage model with evolving internal length. International journal for numerical and analytical methods in geomechanics, 28(7-8):633-652, 2004.

G. Pijaudier-Cabot, A. Krayani, F. Dufour, H. Yuan, and F. Wittmann. Comments on boundary effects in non local damage based models. Nonlocal modelling of failure of materials. Aedificatio Pubs, 2007.

G. Pijaudier-Cabot, F. Dufour, and M. Choinska. Permeability due to the increase of damage in concrete: from diffuse to localized damage distributions. Journal of engineering mechanics, 135(9):1022-1028, 2009.

F. Ragueneau, R. Desmorat, and F. Gatuingt. Anisotropic damage modelling of biaxial behaviour and rupture of concrete structures. Computers $\&$ Concrete, 5(4):417-434, 2008.

G. Rastiello, C. Boulay, S. Dal Pont, J. Tailhan, and P. Rossi. Real-time water permeability evolution of a localized crack in concrete under loading. Cement and Concrete Research, 56:20-28, feb 2014. ISSN 00088846. doi: 10.1016/j.cemconres.2013. 09.010. URL http://www.sciencedirect.com/science/article/pii/S0008884613001968.

G. Rastiello, J.-L. Tailhan, P. Rossi, and S. Dal Pont. Macroscopic probabilistic cracking approach for the numerical modelling of fluid leakage in concrete. Annals of Solid and Structural Mechanics, pages 1-16, 2015. ISSN 1867-6936. doi: 10.1007/s12356-015-0038-6. URL http://link.springer.com/10.1007/s12356-015-0038-6http://dx.doi.org/10.1007/ s12356-015-0038-6.

G. Rastiello, C. Desmettre, J.-L. Tailhan, P. Rossi, J.-P. Charron, and S. Dal Pont. Modeling of fluid leakage through multi-cracked RC structural elements using a numerical probabilistic cracking approach. Materials and Structures, 49(8): 3095-3108, 2016. ISSN 1871-6873. doi: 10.1617/s11527-015-0706-3. URL http://dx.doi.org/10.1617/s11527-015-0706-3.

E. Rouy and A. Tourin. A viscosity solutions approach to shape-from-shading. SIAM Journal on Numerical Analysis, 29(3): 867-884, 1992. ISSN 00361429. URL http://www.jstor.org/stable/2158283.

S. Saroukhani, R. Vafadari, and A. Simone. A simplified implementation of a gradient-enhanced damage model with transient length scale effects. Computational Mechanics, 51(6):899-909, 2013.

S. Secchi and B. Schrefler. A method for 3-d hydraulic fracturing simulation. International journal of fracture, pages 1-14, 2012 .

J. a. Sethian. A fast marching level set method for monotonically advancing fronts. Proceedings of the National Academy of Sciences of the United States of America, 93(4):1591-1595, 1996. ISSN 00278424. doi: 10.1073/pnas.93.4.1591.

J. a. Sethian. Fast Marching Methods. SIAM Review, 41(2):199-235, 1999. ISSN 0036-1445. doi: 10.1137/S0036144598347059.

A. Simone, G. N. Wells, and L. J. Sluys. From continuous to discontinuous failure in a gradient-enhanced continuum damage model. Computer Methods in Applied Mechanics and Engineering, 192(41):4581-4607, 2003.

E. Tamayo-Mas and A. Rodríguez-Ferran. A new continuous-discontinuous damage model: Cohesive cracks via an accurate energy-transfer process. Theoretical and Applied Fracture Mechanics, 69(Supplement C):90 - 101, 2014. ISSN 0167-8442. doi: https://doi.org/10.1016/j.tafmec.2013.11.009. URL http://www.sciencedirect.com/science/article/ pii/S0167844213000785. Introducing the new features of Theoretical and Applied Fracture Mechanics through the scientific expertise of the Editorial Board.

F. van der Meer and L. Sluys. The Thick Level Set method: Sliding deformations and damage initiation. Computer Methods in Applied Mechanics and Engineering, 285:64-82, mar 2015. ISSN 00457825. doi: 10.1016/j.cma.2014.10.020. URL http://www.sciencedirect.com/science/article/pii/S0045782514003909.

H. Zhao. A fast sweeping method for eikonal equations. Mathematics of computation, 74(250):603-627, 2005.

O. Zienkiewicz and R. Taylor. The Finite Element Method: Solid mechanics. Referex collection.Mecánica y materiales. Butterworth-Heinemann, 2000. ISBN 9780750650557. URL https://books.google.fr/books?id=MhgBfMWFVHUC. 